\title{
Imaging and Rapid-Scanning Ion Mass Spectrometer (IRM) for the CASSIOPE e-POP Mission
}

\author{
Andrew W. Yau ${ }^{1}$ - Andrew Howarth ${ }^{1}$ Andrew White ${ }^{1}$. \\ Greg Enno $^{1} \cdot$ Peter Amerl $^{1}$
}

Received: 3 December 2014 / Accepted: 30 March 2015 / Published online: 15 April 2015

(C) The Author(s) 2015. This article is published with open access at Springerlink.com

\begin{abstract}
The imaging and rapid-scanning ion mass spectrometer (IRM) is part of the Enhanced Polar Outflow Probe (e-POP) instrument suite on the Canadian CASSIOPE small satellite. Designed to measure the composition and detailed velocity distributions of ions in the $\sim 1-100 \mathrm{eV} / \mathrm{q}$ range on a non-spinning spacecraft, the IRM sensor consists of a planar entrance aperture, a pair of electrostatic deflectors, a time-of-flight (TOF) gate, a hemispherical electrostatic analyzer, and a micro-channel plate (MCP) detector. The TOF gate measures the transit time of each detected ion inside the sensor. The hemispherical analyzer disperses incident ions by their energy-per-charge and azimuth in the aperture plane onto the detector. The two electrostatic deflectors may be optionally programmed to step through a sequence of deflector voltages, to deflect ions of different incident elevation out of the aperture plane and energy-per-charge into the sensor aperture for sampling. The position and time of arrival of each detected ion at the detector are measured, to produce an image of 2-dimensional (2D), mass-resolved ion velocity distribution up to 100 times per second, or to construct a composite 3D velocity distribution by combining successive images in a deflector voltage sequence. The measured distributions are then used to investigate ion composition, density, drift velocity and temperature in polar ion outflows and related acceleration and transport processes in the topside ionosphere.
\end{abstract}

Keywords Ion mass spectrometer · Time of flight · Electrostatic analyzer · Ion outflows

\section{Introduction}

As previously anticipated in Chappell (1988) and recently observed on the Cluster satellites (Engwall et al. 2009), the magnetosphere often contains a significant component of "cold" ions originating as ion outflows from the ionosphere. In the polar ionosphere, both ion density and mass composition are important parameters in ionospheric ion acceleration and outflow. For example, the ion mass density affects the properties of Alfven waves, which carry

\footnotetext{
$凶$ A.W. Yau

yau@ucalgary.ca

1 Department of Physics and Astronomy, University of Calgary, Calgary, Alberta, Canada
} 
field-aligned currents and plasma waves (Lysak and Lotko 1996), and the plasma density is believed to control auroral acceleration and auroral kilometric radiation (AKR) (Morooka and Mukai 2003). The ion convection velocity is also an important plasma parameter, due to its direct $(\mathbf{E} \times \mathbf{B})$ relationship with the convection electric field in the "frozen-in" and collision-less regions of the magnetosphere and the ionosphere, where large-scale convective electric fields play a critical role in plasma circulation, redistribution and energization.

Many of the existing techniques for measuring thermal ions are based on electrostatic or retarding potential analysis, and focused on resolving the energy and angular distributions of the measured ions. Some of these techniques also resolve the mass composition of the measured ions. A retarding potential analyzer (RPA) sweeps its retarding potential grid through a range of potentials, to generate a characteristic curve of the measured ion current as a function of the retarding potential (Hanson et al. 1977; Coley et al. 2010), and the RPA curve is typically fitted to a model to infer the density, velocity, temperature, and under certain conditions the relative ion composition of the measured ions.

The earlier particle-counting ion energy analyzers typically used channel electron multipliers, and measured ions at one energy or retarding potential and one incident direction at a time. Likewise, the mass-resolving analyzers typically sampled one or a small number of mass species at a time. The Dynamics Explorer-1 (DE-1) retarding ion mass spectrometer (RIMS; Chappell et al. 1981), which used a sector magnet behind a RPA to simultaneously sample two mass steps of a fixed ratio, and the Akebono suprathermal ion mass spectrometer (SMS; Whalen et al. 1990), which combined a modified Bennett RF ion mass spectrometer (Bennett 1950), retarding potential and electrostatic analyzers, and a micro-channel plate (MCP) detector for limited ion energy or angular imaging (Yau et al. 1998a), are notable examples.

The advent of the MCP detector has made possible the design of "imaging" plasma analyzers capable of resolving the angular, energy, or mass distribution of the measured ions or electrons in a single measurement. Carlson et al. (1983) pioneered the top-hat analyzer design, which allows the simultaneous sampling of particles at a particular energy-per-charge over the full $360^{\circ}$ range of incident azimuth, thus providing an effective means to image ions or electrons (Pollock et al. 1998) over the full $180^{\circ}$ range of pitch-angle when the axis of the analyzer is oriented perpendicular to the local magnetic field.

In contrast with the top-hat design, the hemispherical electrostatic analyzer (HEA) design (Whalen et al. 1994) allows the simultaneous sampling of particles over not only the full $360^{\circ}$ range of incident azimuth but also an extended range of energy-per-charge. A timeof-flight gate was added to this design in the thermal plasma analyzer (TPA) on Nozomi (Yau et al. 1998b), giving the analyzer a capability to image the mass-resolved velocity distribution of each ion species in two dimensions (2D). On the spinning Nozomi spacecraft, successive 2D distributions combined over half a spacecraft spin period results in obtaining the corresponding 3-dimensional (3D) distribution.

The scientific objective of the Enhanced Polar Outflow Probe (e-POP) payload is to make observations of mesoscale and microscale plasma processes in the topside high-latitude ionosphere at the highest-possible resolution, specifically to study the microscale characteristics of plasma outflow and related acceleration processes, the occurrence morphology of neutral escape, and the effects of auroral currents on plasma outflow and those of plasma microstructures on radio propagation.

The variety of observed ion outflows in the high-latitude ionosphere may be grouped into two categories: bulk ion flows with energies up to a few $\mathrm{eV}$ in which all the ions acquire a bulk flow velocity, and suprathermal ion outflows in which in general a fraction of the ions are energized to much higher energies. The category of bulk ion flows includes the polar 
wind and auroral bulk $\mathrm{O}^{+}$up-flow from the topside auroral and polar-cap ionosphere. The category of suprathermal ion outflows includes ion beams, ion conics, transversely accelerated ions (TAI), and upwelling ions (UWI).

A number of acceleration mechanisms have been proposed for the observed ion up-flows and outflows (see e.g. Strangeway et al. 2005), including direct heating of ions by plasma waves (Andre et al. 1998), frictional heating and subsequent mirroring, and ambipolar outflow driven by photoelectrons (Abe et al. 1993) or by heated ionospheric electrons resulting from soft auroral precipitating electrons (Redmon et al. 2012).

In order to observe the key parameters relevant to ion up-flows and outflows in the topside ionosphere and their drivers, the e-POP payload includes the imaging and rapid-scanning ion mass spectrometer (IRM) to measure the low-energy ion population, in addition to a suprathermal electron imager (SEI) to measure selectively the low-energy electron or ion energy distributions (Knudsen et al. 2015; this issue), a magnetic field instrument (MGF) to characterize the field-aligned current systems (Wallis et al. 2014; this issue), a plasma wave receiver (RRI) to observe the in-situ plasma wave environment in the VLF and HF frequency range (James et al. 2014; this issue), a fast auroral imager (FAI) to provide the auroral context (Cogger et al. 2014; this issue), and a neutral mass spectrometer (NMS) to characterize the neutral population.

The basic measurement objective of IRM is to resolve the mass composition and to measure the velocity distributions of thermal and suprathermal energy ions in the energy-percharge range of $\sim 1$ to $100 \mathrm{eV} / \mathrm{q}$ and the mass-per-charge range from 1 to 40 atomic mass units per charge (AMU/q), and to infer from the measured distributions the ion composition, density, drift velocity and temperature. An important element of the objective is to resolve the major constituents $\left(\mathrm{H}^{+}, \mathrm{He}^{+}\right.$and $\left.\mathrm{O}^{+}\right)$and under certain conditions, the minor ion species in the topside ionosphere, including molecular ions such as $\mathrm{N}_{2}^{+}, \mathrm{NO}^{+}$and $\mathrm{O}_{2}^{+}$.

\section{Instrument Design and Principle of Operation}

The IRM sensor consists of a planar entrance aperture, a pair of electrostatic deflectors, a time-of-flight (TOF) gate, a hemispherical electrostatic analyzer (HEA), and a MCP detector. It is housed in a cylindrical enclosure and mounted onto the end of an aluminum boom on the CASSIOPE spacecraft. Figure 1a depicts the placement, orientation, and field-ofview (FOV) of the IRM sensor on the spacecraft. When CASSIOPE is in the ram-pointing mode, the spacecraft coordinate system $+\mathrm{X}$-axis is along the ram direction, and the $+\mathrm{Z}$-axis is towards nadir. The IRM boom is mounted on the " $-\mathrm{Y}$ " side (right side in the anti-ram direction) of the spacecraft so that its entrance aperture plane is along the X-Z ("nadir-ram") plane of the spacecraft with a $360^{\circ} \mathrm{FOV}$ and at a distance of $0.95 \mathrm{~m}$ from the spacecraft surface. As will be discussed below, the sensor FOV out of the aperture plane is controlled by the electrostatic deflectors.

Figure $1 \mathrm{~b}$ is a schematic cross section of the sensor through its rotational axis, and it shows the sensor's entrance aperture plane, the pair of deflection rings that form the toroidal electrostatic deflector, the TOF gates, the inner and the outer hemispherical electrostatic domes that form the HEA, the MCP, and the detector anode printed circuit board. Figure 2 identifies the bias voltages at the deflection rings, the hemispherical domes, the TOF gate, as well as other voltage grids inside the sensor, and Table 1 lists the respective voltage values or ranges.

The ion optics of the sensor is rotationally symmetric and is defined principally by the sensor's planar entrance aperture, toroidal electrostatic deflector and HEA. The top and bottom deflection rings can be biased to selected voltages of opposite polarity $\left(V_{E D}+\right.$ and 


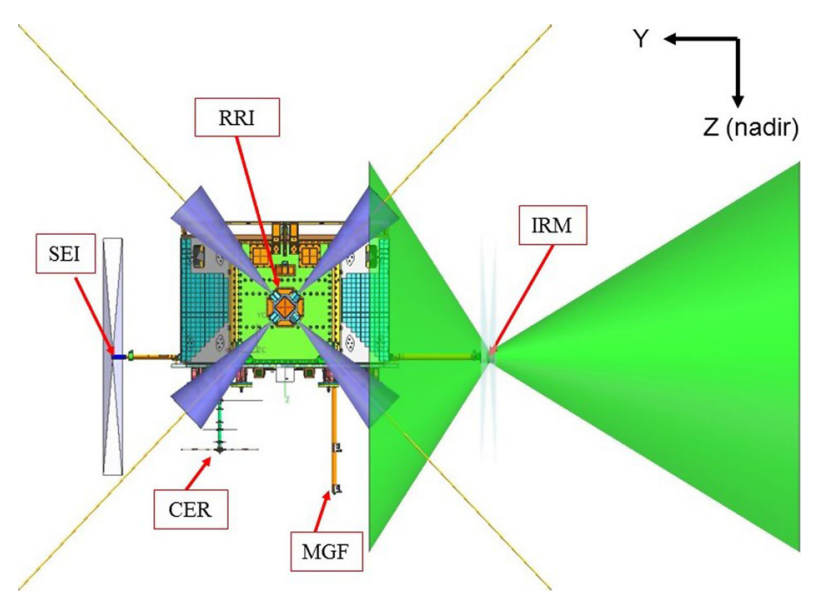

Fig. 1a Schematic view of IRM sensor placement, orientation, and field-of-view (FOV) in the spacecraft $\mathrm{X}-\mathrm{Z}$ plane, showing the $360^{\circ} \mathrm{FOV}$ in azimuth (in the sensor aperture plane), $\pm 2^{\circ}$ elevation angle of acceptance (out of the aperture plane; between the light blue triangles), and the sampled range of clear FOV in elevation angle $\left(-30^{\circ}\right.$ to $+45^{\circ}$; between the green triangles). The $+\mathrm{Z}$ axis points toward nadir; the $+\mathrm{X}$ axis points toward ram. SEI = suprathermal electron imager, MGF = magnetic field instrument, RRI $=$ radio receiver instrument, $\mathrm{CER}=$ coherent electromagnetic radio tomography instrument on e-POP

Fig. 1b Schematic cross section of e-POP IRM sensor in the spacecraft $\mathrm{X}-\mathrm{Y}$ plane

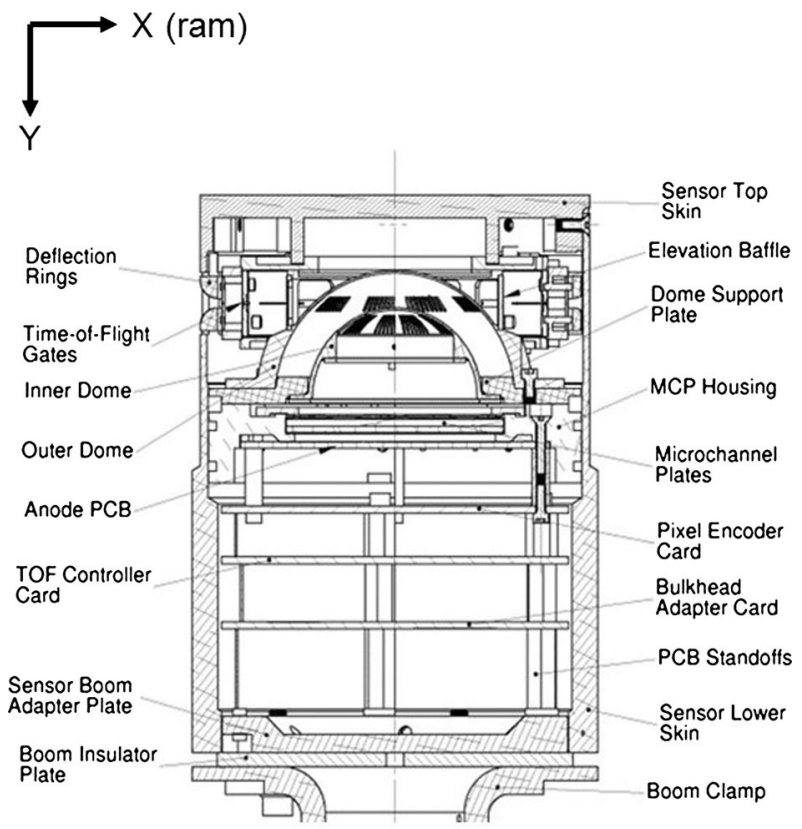

$V_{E D^{-}}$), to deflect incident ions of specific energy-per-charge and elevation combinations into the TOF gate. The TOF gate opens and closes repeatedly to control ion entry in a measurement, by using a pair of fast-switching electrodes that are controlled by the TOF gate driver. Both the sensor entrance and the outer dome are biased to the sensor high voltage (HV) ground (i.e. $V_{E A}=V_{E S}=0$ ). The inner dome is biased negative relative to the 
Table 1 IRM sensor bias voltages

\begin{tabular}{lll}
\hline Voltage & Definition & Allowed value/range (V) \\
\hline$V_{E D}$ & Toroidal electrostatic deflector; \pm at top and bottom rings & -10 to +10 \\
$V_{E S}$ & Hemispherical electrostatic analyzer (HEA) outer dome & 0 \\
$V_{E A}$ & Entrance aperture bias & 0 \\
$V_{T F}$ & Time-of-flight gate electrode (bottom electrode) & +10 \\
$V_{S A}$ & Hemispherical electrostatic analyzer (HEA) inner dome & 0 to -353 \\
$V_{F P}$ & Micro-channel plate (MCP) front surface bias & -4 to -2487 \\
$V_{B P}$ & Micro-channel plate (MCP) back surface bias & 0 to -356 \\
$V_{N C}$ & Anode & 0 \\
\hline
\end{tabular}

Fig. 2 Sensor bias voltages defined in Table 1

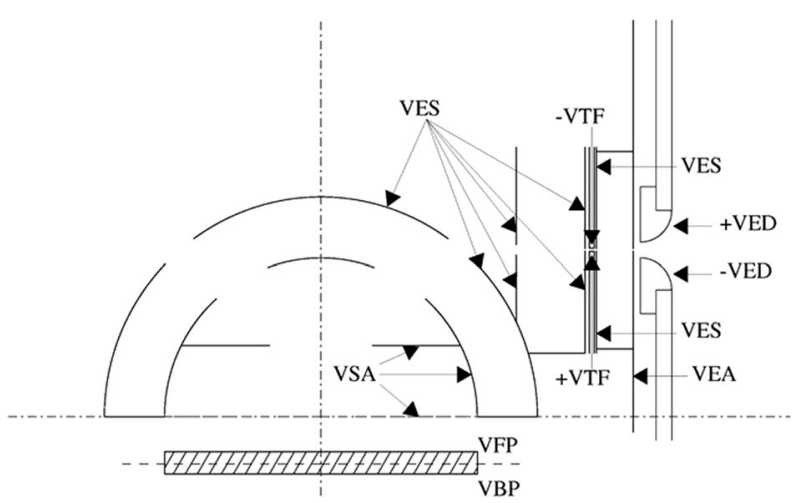

outer dome $\left(V_{S A}<V_{E S}\right)$. The resulting central electric field disperses incident ions by their energy-per-charge and azimuth onto the MCP detector. The landing radius and azimuth of each detected ion on the MCP surface maps to the ion energy and azimuth of arrival.

To characterize the ion optics of the toroidal electrostatic deflector and the HEA, particletracing computer code was used to calculate the electric field distribution inside the sensor and in the vicinity of its entrance aperture as a function of sensor voltages, and to simulate the ion trajectories inside the sensor as a function of incident ion energy-per-charge, massper-charge, elevation and azimuth. Figure 3 shows the simulated trajectories of incident ions of 4-60 eV/q energy-per-charge in the sensor, in response to a bias voltage of $V_{S A}=$ $-200 \mathrm{~V}$ in the HEA and $V_{E D \pm}=\mp 1.2 \mathrm{~V}$ at the top and bottom deflector ring, respectively. The deflector acts as an energy-elevation selector by accepting lower-energy ions at larger incident elevation and higher-energy ions at smaller elevation. Note that when both the top and bottom deflection plates are set to ground, the sensor samples only ions at $0( \pm 2)^{\circ}$ elevation, and measures the $2 \mathrm{D}$ velocity distribution in the entrance aperture plane.

The HEA disperses ions passing through the TOF gate by their energy-per-charge and azimuth, by focusing ions of a given azimuth and energy-per-charge onto a point at the opposite azimuth on its hemispherical plane, and at a radial distance that increases approximately with the square of ion energy-per-charge for a given voltage difference between $V_{S A}$ and $V_{E S}$, essentially irrespective of the elevation angles of the ions. Thus, the highest energy ions arrive at the outermost portion of the detector, and the energy range of the detected ions depends primarily on $\Delta V_{S A} ; \Delta V_{S A}=V_{S A}-V_{E S}$. 


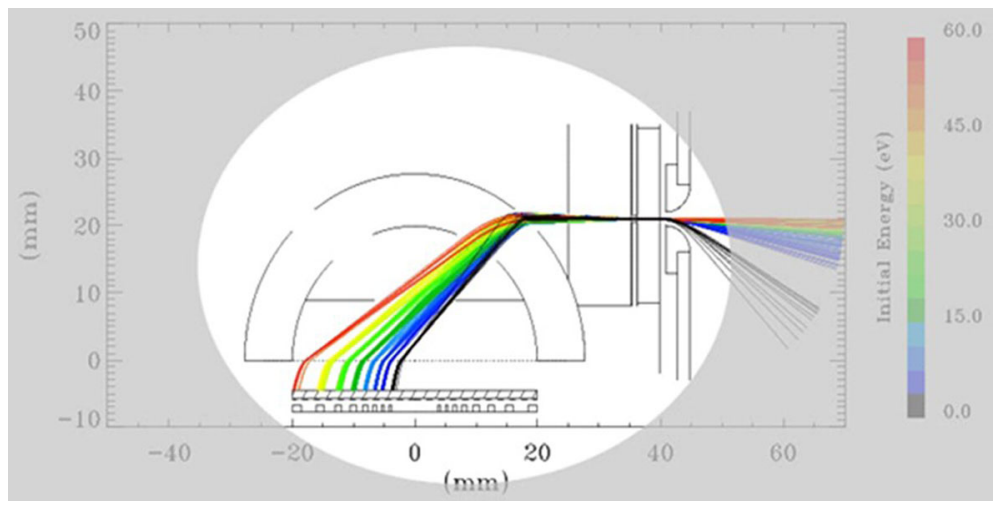

Fig. 3 Ion optics of the toroidal electrostatic deflector and hemispherical electrostatic analyzer (HEA): side-view of simulated trajectories of incident ions at $4-60 \mathrm{eV} / \mathrm{q}$ and $0^{\circ}-60^{\circ}$ elevation, in the presence of voltage bias $V_{E D \pm}$ of $-1.2 \mathrm{~V}$ and $+1.2 \mathrm{~V}$ at the top and bottom deflection ring, respectively, and $V_{S A}$ of $-200 \mathrm{~V}$ at the inner dome, with the smaller-energy ions being deflected the most in elevation and arriving at the innermost part of the MCP detector

Fig. 4 Top: Time-of-flight (TOF) gate open-and-close sequence in a TOF cycle; bottom: Multiple TOF cycles of TOF gate open and close in a measurement
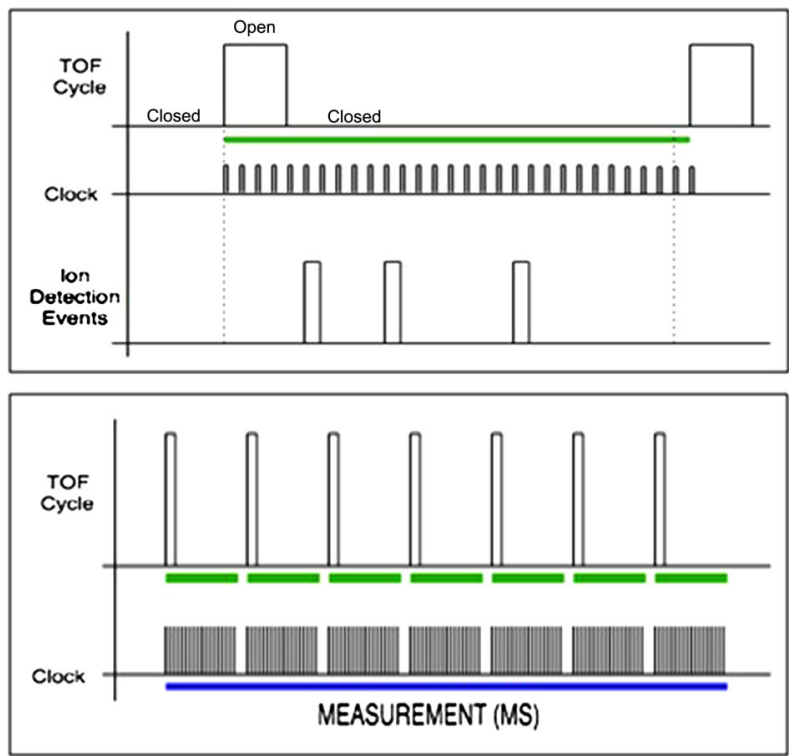

Figure 4 depicts the operation of the TOF gate, by showing its multiple TOF cycles of gate open and close sequence in each measurement. Each TOF cycle is $40.96 \mu$ s in duration, and consists of 1024 TOF bins of $40 \mathrm{~ns}$ each in duration $\left(N_{B I N}=1024 ; T_{B I N}=40 \mathrm{~ns}\right)$. A measurement consists of a number of TOF cycles, $N_{T O F}$, which can range from 1 to 65535 and has a default value of 240 , corresponding to a range of measurement period from $40.96 \mu$ s to $2.56 \mathrm{~s}$ and a default measurement period of $9.83 \mathrm{~ms}$ (i.e. 100 measurements per second). In each TOF cycle, the TOF gate can remain open for up to 255 TOF bins $\left(N_{O P N}=0-255\right) ; N_{O P N}$ is typically between 10 and 50 , corresponding to a duty cycle of $1-5 \%$ and a gate-open period of 0.4 to $2 \mu \mathrm{s}$. 
The detector consists of a pair of MCPs and a discrete anode. The front side of the anode contains 64 discrete pixels, arranged in 8 angular pixel sectors of 8 energy pixels each. An energy pixel in a specific pixel sector will be referred to as a pixel, detector pixel, or energy-angle pixel interchangeably, and the innermost (lowest) and the outermost (highest) energy pixel as pixel 1 and 8, respectively, below. An incident ion passing through the HEA produces a charge as it arrives at the front surface of the MCP. This charge is amplified into a "charge cloud" through successive collisions with the channel walls inside the MCP. The charge cloud is proximity focused at the back of the MCP and then slightly defocused before being collected onto the anode; the defocusing is to increase the spatial spread of the cloud, from tens of microns to hundreds of microns.

On the backside of the detector anode, each pixel is connected to a pair of charge amplifiers, each of which detects the charge buildup at the pixel (a "pixel hit") above a threshold value $\left(2.9 \times 10^{5}\right.$ electrons $)$. This reduces the number of amplifiers needed from 64 to 16 . In order to direct roughly half of the charge hitting a pixel to each of the two charged amplifiers, each pixel is subdivided into two interlaced, separated and electrically isolated halves. Each half is connected to one of the two amplifiers, so that statistically each amplifier is likely to detect half of the charge cloud. The purpose of the charge defocusing above is to distribute the charge cloud over a larger fraction of a pixel's surface area, so as to split the charge more evenly between the two charge amplifiers. A consequence of the interlaced pixel subdivision is that whenever the split of a charge cloud is very unequal between the two amplifiers the charge may be detected above the threshold level at only one of the two amplifiers. When this occurs, the event is recorded as a "pixel detect" as opposed to a "pixel hit".

On the pixel encoder card, the circuitry of pre-amplifiers identifies the energy-angle pixel (address) of each detected ion, and the TOF bin corresponding to the ion arrival time relative to the TOF gate opening. A field programmable gate array (FPGA) transmits the 6-bit pixel address and 10-bit TOF bin number as a 2-byte data word to a first-in-first-out (FIFO) data buffer, for subsequent determination of the energy-per-charge, mass-per-charge, and incident azimuth and elevation of each detected ion based on the fixed sensor voltage settings in a measurement. In addition, the FPGA tracks the number of FIFO data words in a measurement, as "pixel hits" with definitive energy-angle pixel address, and the number of ions detected regardless of their pixel address, such as those detected by only one preamplifier, as "pixel detects".

Figure 3 shows that for a given sensor voltage setting, each of the 8 energy pixels in each pixel sector responds to incident ions of a specific, narrow energy range. Figure 5 shows the simulated peak energy-per-charge of ions arriving at each pixel as a function of the pixel radius for different values of $\Delta V_{S A}$, in a log-log plot; $\Delta V_{S A}=V_{S A}-V_{E S} ; V_{E S}=0$, and $V_{F P}=-1950 \mathrm{~V}$.

To a first approximation, the peak ion energy-per-charge varies as the square of the pixel radius. For a given $\Delta V_{S A}$, the peak energy-per-charge ranges from $\sim 0.005-0.01 \Delta V_{S A}$ for the innermost (lowest-energy) pixel to $\sim 0.25-0.30 \Delta V_{S A}$ for the outermost (highest-energy) pixel; it increases slightly with the magnitude of the MCP front surface bias, $V_{F P}$, by $<2 \%$ for an increase of $200 \mathrm{~V}$ in $\left|V_{F P}\right|$. Thus, the peak energy ratio between the highest and lowest energy pixels is $\sim 20$ at $\Delta V_{S A}$ of $20 \mathrm{~V}, \sim 47$ at $\Delta V_{S A}$ of $350 \mathrm{~V}$.

As shown in Whalen et al. (1994), the finite range of the ion entrance position in the aperture plane results in a small inward spread in the radial focusing $(\sim 10 \%)$ and a symmetric spread in the azimuth focusing $\left(\sim 5^{\circ}\right.$ to $\left.15^{\circ}\right)$, as incident ions near the edge of the sensor are deflected to a slightly smaller radius for a given incident ion energy and azimuth. The detector pixel widths were chosen to optimize the ion energy and angular sampling range and resolution given this de-focusing, and to minimize the differences in size between pixels and the resulting potential amplifier crosstalk, while keeping the design and machining 
Fig. 5 Peak ion energy per charge as a function of the detector pixel radius at selected $V_{S A}$ settings

Fig. 6 Mass response of the IRM sensor: Time of flight (TOF, in units of TOF bin number; $1 \mathrm{TOF}$ bin period $=40 \mathrm{~ns}$ ) as a function of ion energy-per-charge in each detector pixel, for $\mathrm{H}^{+}$, $\mathrm{He}^{+}, \mathrm{N}^{+}, \mathrm{O}^{+}$, and $\mathrm{NO}^{+}$, respectively, for the case of $V_{S A}=-149 \mathrm{~V}$ (solid curves), and for $\mathrm{H}^{+}$and $\mathrm{O}^{+}$only for the case of $V_{S A}=-64 \mathrm{~V}$ (dotted curves) and $V_{S A}=-350 \mathrm{~V}$ (dashed curves), respectively
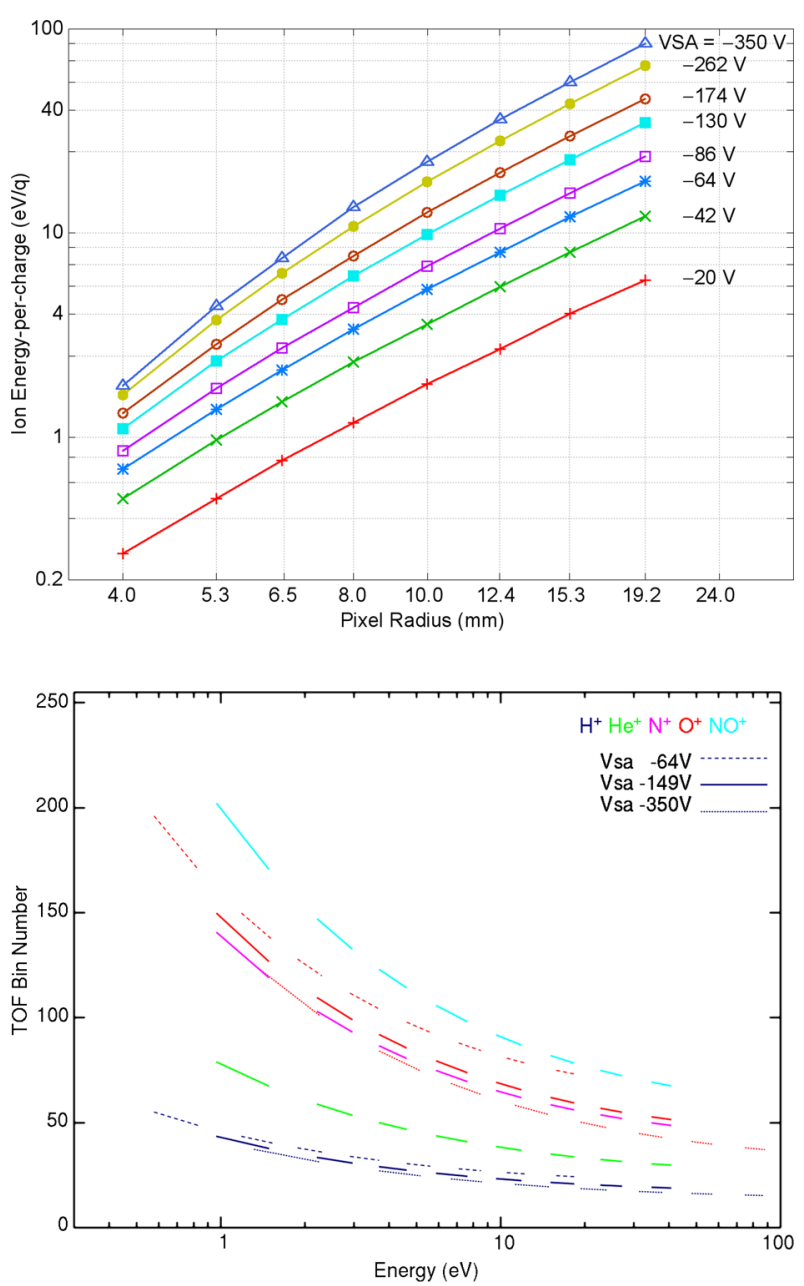

tolerance requirements to an achievable level. The radial width $\Delta r$ ranges from $0.33 \mathrm{~mm}$ $(\Delta r / r=0.075)$ for the innermost pixel to $1.6 \mathrm{~mm}(\Delta r / r=0.042)$ for the outermost pixel; the corresponding full energy width at half maximum (FWHM) $\Delta E / E$ ranges from 15$24 \%$ to $5-7 \%$. The corresponding angular width $\Delta \phi$ ranges from $40^{\circ}$ to $3^{\circ}$; cf. Fig. 7 below.

Figure 6 shows the ion TOF as a function of ion energy-per-charge in each energy pixel, for the five most abundant or common ion species $\left(\mathrm{H}^{+}, \mathrm{He}^{+}, \mathrm{N}^{+}, \mathrm{O}^{+}\right.$, and $\mathrm{NO}^{+}$, respectively, in color code) in the topside ionosphere, in TOF bin number (1 TOF bin period $=40 \mathrm{~ns}$ ) for the case of $V_{S A}=-149 \mathrm{~V}$, and for $\mathrm{H}^{+}$and $\mathrm{O}^{+}$only for the case of $V_{S A}=-64 \mathrm{~V}$ and $-350 \mathrm{~V}$, respectively. Figure 6 shows that for each $V_{S A}$ and within each pixel, the TOF ranges of the different ion species are well separated from each other, with the heaviest ion species having the largest TOF values. For each ion species the range of TOF sampled decreases with increasing $V_{S A}$ both within a pixel and over all pixels. Within each pixel, the TOF decreases with increasing ion energy for a given $V_{S A}$, and the smallest (largest) TOF value corresponds to ions of maximum (minimum) energy-per-charge at the pixel. 


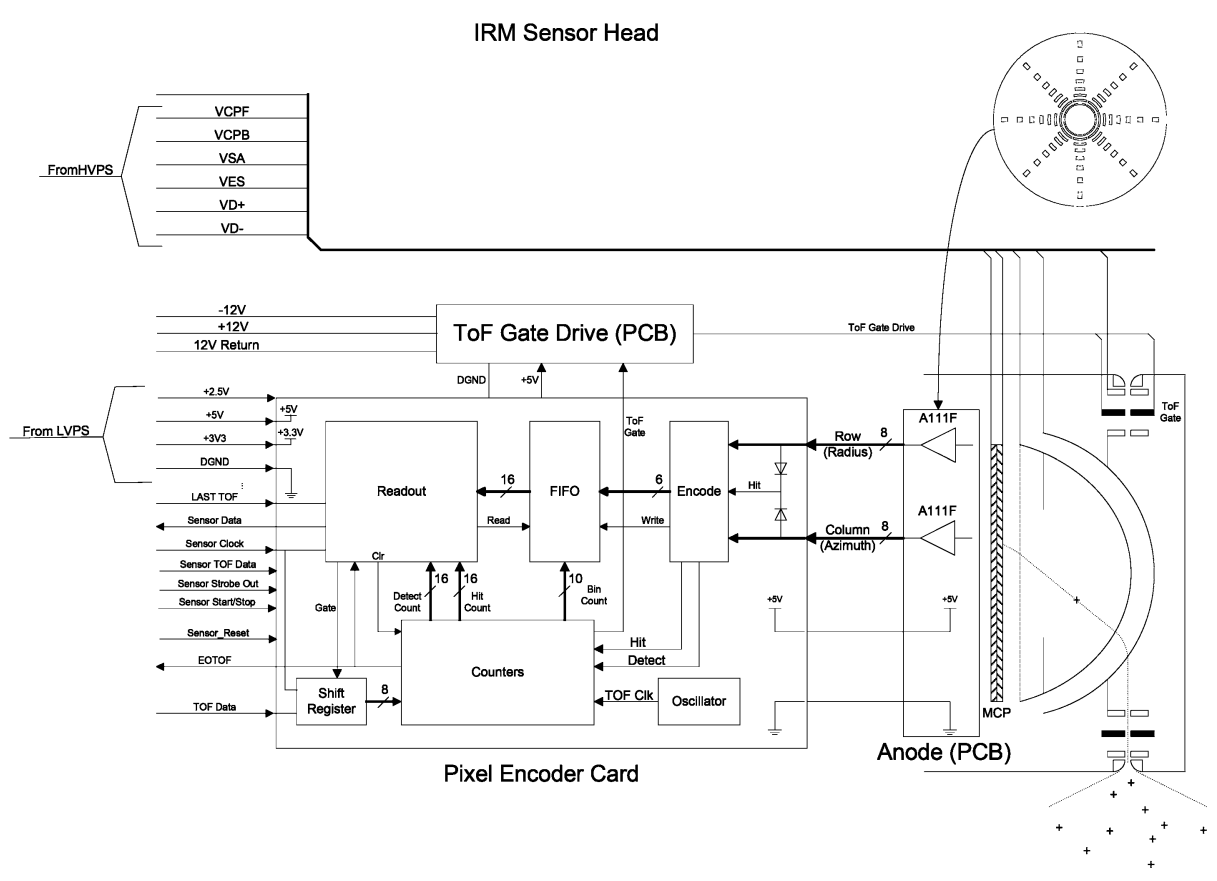

Fig. 7 IRM sensor block diagram

As noted above, the TOF gate remains open for $N_{O P N}$ TOF bins at the beginning of each TOF cycle. The TOF in Fig. 6 therefore corresponds to the arrival time of ions of normal incidence that are at the TOF gate at the beginning of a TOF cycle. Ions arriving at the gate later during the TOF gate-open period and off-normal incidence ions have slightly later arrival times, which appear as larger TOF values. Note also that $V_{F P}=-2030 \mathrm{~V}$ was used in Fig. 6, and that as can be inferred from Fig. 5 above, the value of TOF decreases negligibly with increasing magnitude of $V_{F P}\left(<1 \%\right.$ over the operational range of $\left.V_{F P}\right)$.

Figure 6 shows that the sensor can sample only a small fraction $(25-40 \%)$ of the ion energy range between $\sim 0.3$ and $90 \mathrm{eV}$ at a fixed $V_{S A}$ setting. However, by stepping through multiple $V_{S A}$ steps, it can sample essentially the full energy range, and moreover, sample ions at a particular energy at different angular resolution, by taking advantage of the different angular widths of the different energy pixels in each pixel sector.

Figure 7 shows a schematic block diagram for the sensor, including the architecture of the TOF gate driver and anode printed circuit boards, the pixel encoder card, and the series of high and low voltage grids. Figure 8 shows a schematic block diagram of the electronics module, which consists of a digital signal processor (DSP), a low voltage power supply (LVPS), and a high voltage power supply (HVPS). The DSP acts as the command and data interface with the spacecraft, and controls the operations of both the HVPS and the sensor. The HVPS provides the sensor voltages and their ground reference. The LVPS converts the spacecraft bus power (+28 V DC) into regulated and isolated $+3.3 \mathrm{~V} \mathrm{DC},+5 \mathrm{~V} \mathrm{DC}$ and \pm 12 V DC outputs.

The cylindrical sensor enclosure is mated onto the end of an aluminum boom, as noted above, with polysulfone. The body of the boom is electrically connected to the spacecraft ground, and the multi-layer insulation (MLI) material covering the boom is coated with a film of indium tin oxide (ITO), to provide a finite potential gradient between the sensor 


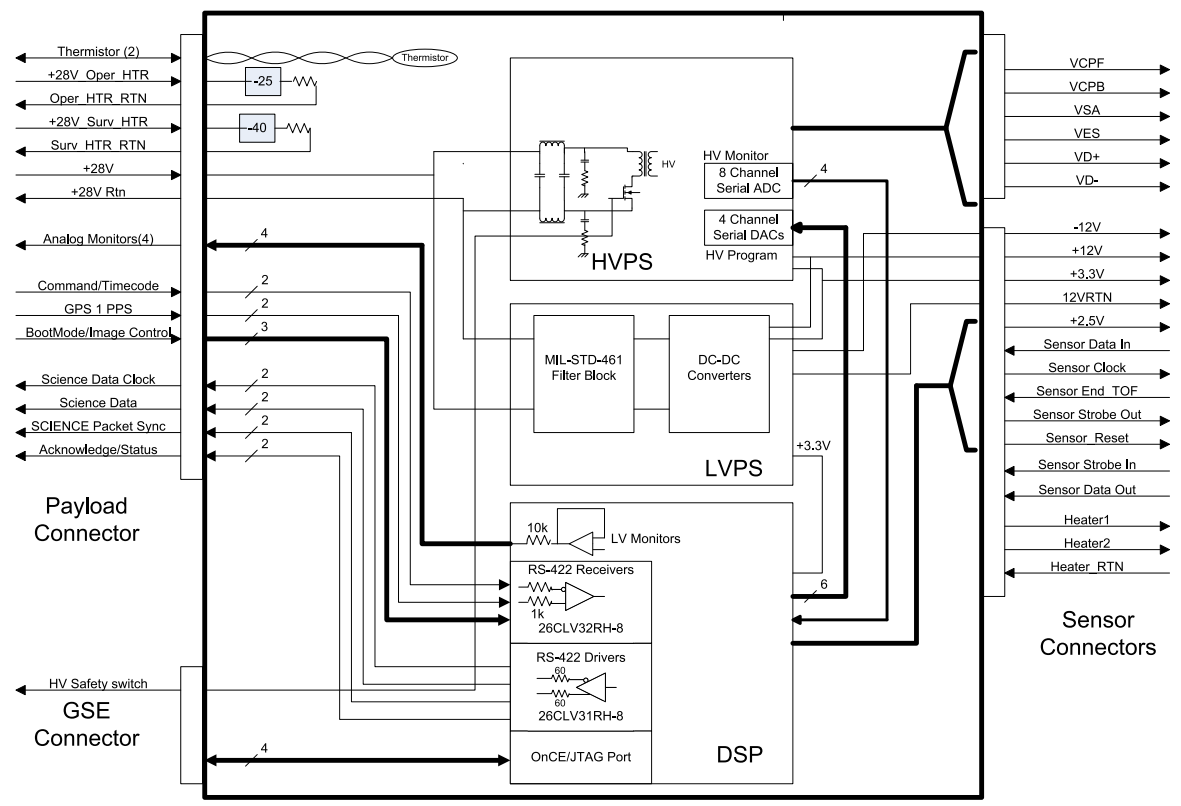

Fig. 8 IRM electronics module block diagram

and spacecraft surfaces, and to allow the sensor surface to float at its own potential $\phi_{s}$ with respect to the ionosphere.

Both the top and the cylindrical surfaces are coated with a conductive paint (RM-400 from AZ Technologies, with a surface conductivity of $\sim 1.0 \mathrm{M} \Omega$ /square), and electrically connected to sensor ground, to provide a measure of the incident current on the sensor surfaces. In general, this current consists of contributions from the ambient and non-ambient electrons and ions, respectively, including photoelectrons emitted from the sensor surface, i.e. $I=I_{a i}+I_{a e}+I_{n i}+I_{n e}$, where the subscripts $a, n, i$, and $e$ denote ambient, non-ambient, ion, and electron. Based on the surface areas of the two surfaces $\left(A_{T}=64 \mathrm{~cm}^{2}, A_{C}=\right.$ $353 \mathrm{~cm}^{2}$ ), the measured current $I$ is expected to range from $\sim-10 \mu \mathrm{A}$ to a few $\mu \mathrm{A}$ at orbit altitudes under typical ionospheric conditions outside of regions of intense energetic electron precipitation, and may thus provide a monitor of the ionospheric electron density and temperature under certain assumptions and spacecraft conditions.

\section{Sensor Response}

To characterize the sensor mass (TOF), energy, angular, and sensitivity responses pre-flight calibration measurements were made using two low-energy (1-200 eV) ion sources: one with a beam diameter of $\sim 2 \mathrm{~cm}$ and beam width of $\pm 2^{\circ}$ and the other with a diameter of $\sim 10 \mathrm{~cm}$ and a higher output beam current up to $1 \mathrm{nA}$. A Faraday cup was used for absolute flux calibration and for beam energy and width characterization. Both the sensor and the Faraday cup were mounted on a manipulator table, for rotation in 2 axes and translation in 2 directions with respect to the ion source.

In each calibration run, the ion source was pre-programmed to select a neutral gas for ionization (molecular nitrogen mostly and argon occasionally), to produce an ion beam at a 
Fig. 9 Deflection in incident elevation of $15-\mathrm{eV} \mathrm{N}_{2}^{+}$ions as a function of toroidal electrostatic deflector voltage

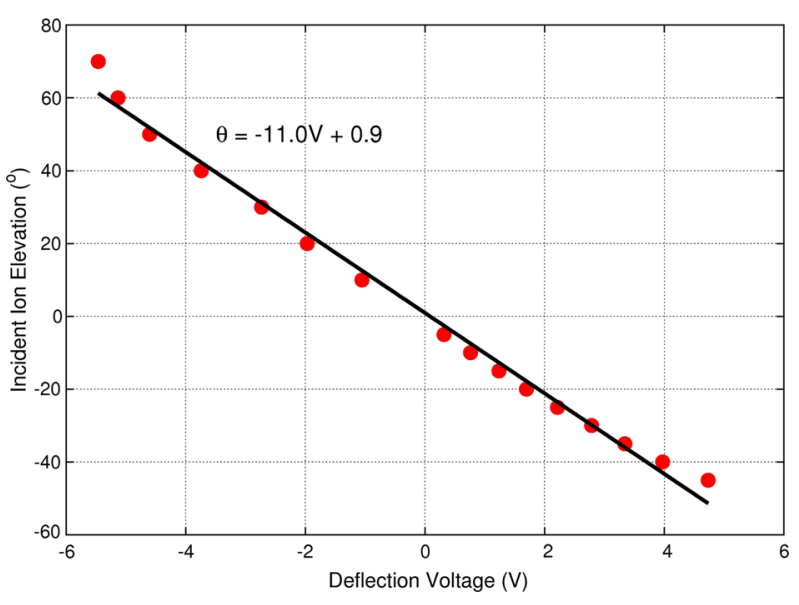

fixed energy, with FWHM of $\sim 30-50 \%$. The majority of calibration runs were performed using ions between 10 and $50 \mathrm{eV}$, which in the case of the $\mathrm{N}_{2}^{+}$ions corresponds to a velocity between 8.3 and $18.6 \mathrm{~km} / \mathrm{s}$, to mimic the ram velocities $(\sim 8 \mathrm{~km} / \mathrm{s})$ of ambient ions and the larger velocities of accelerated ions expected in orbit.

In all calibration runs, the sensor performed measurements repeatedly and continuously with the default measurement period $(9.83 \mathrm{~ms}$; cf. Sect. 2 above). During an azimuth or elevation calibration run, all sensor voltages and TOF gate parameters were kept fixed, while the sensor stepped through a sequence of azimuth or elevation settings with respect to the ion source. During other calibration runs, the sensor azimuth and elevation were fixed, and the sensor stepped through one of the sensor voltages or TOF gate parameters: $V_{S A}, V_{C P}$, and $N_{O P N}$ in the case of energy, sensitivity, and mass calibration runs, respectively.

Figure 9 shows the measured deflection in elevation angle of $15-\mathrm{eV} \mathrm{N}_{2}^{+}$ions as a function of the toroidal electrostatic deflector voltage, using the smaller ion source. The voltage value at each elevation angle in this figure was obtained by measuring the pixel hits in the detector as a function of the varying deflection voltage when the sensor aperture plane was positioned at the elevation angle with respect to the ion source, and fitting the measured profile to a Gaussian.

In Fig. 9, the deflection elevation angle $\theta$ varies linearly with the deflection voltage amplitude $V_{E D}$ except at large elevation angles: i.e. $\theta=\alpha V_{E D}+\beta$ where $\alpha=11.1^{\circ} \mathrm{V}$ and $\beta=$ $0.9^{\circ}$. Such a linear relationship between $\theta$ and $V_{E D}$ was found to hold in calibration runs at other ion energies and runs using argon ions $\left(\mathrm{Ar}^{+}\right)$. In each case, the numerical value of $\alpha$ was found to increase with decreasing incident ion energy, in good accord with simulation; the value of $\beta$ is attributed to the relative misalignment of $\sim 1^{\circ}$ between the sensor and the ion source.

Figure 10 shows two examples of measured TOF spectra of $50 \mathrm{eV} \mathrm{N}_{2}^{+}$ions, using the larger ion source. The top and bottom panels show spectra at 1 and $0.1 \%$ TOF duty cycle, respectively. In both cases, $V_{S A}$ was set to $-300 \mathrm{~V}$. In both cases, two TOF peaks were observed; only one TOF peak was observed in runs using argon. The larger peak starting near TOF bin 54 is attributed to $\mathrm{N}_{2}^{+}$ions, and the smaller peak starting near TOF bin 40 to $\mathrm{N}^{+}$ions. In comparison, it can be inferred from Fig. 6 above that $50-\mathrm{eV}$ ions of 28 and 14 AMU/q $\left(\mathrm{N}_{2}^{+}\right.$and $\left.\mathrm{N}^{+}\right)$are expected to peak starting at TOF bin 53 and 41, respectively.

The TOF bin number of a measured ion is determined by the time delay $\tau$ between the start of the TOF cycle and the time of ion arrival at the detector pixel. This time delay is 
Fig. 10 Measured time-of-flight (TOF) spectra of $50 \mathrm{eV} \mathrm{N}_{2}^{+}$ions: top: $1 \%$ TOF duty cycle (TOF gate open period $=10 \mathrm{TOF}$ bins $=0.4 \mu \mathrm{s}$ ); bottom: $0.1 \% \mathrm{TOF}$ duty cycle (TOF gate open period $=1 \mathrm{TOF}$ bin $=0.04 \mu \mathrm{s}$ )
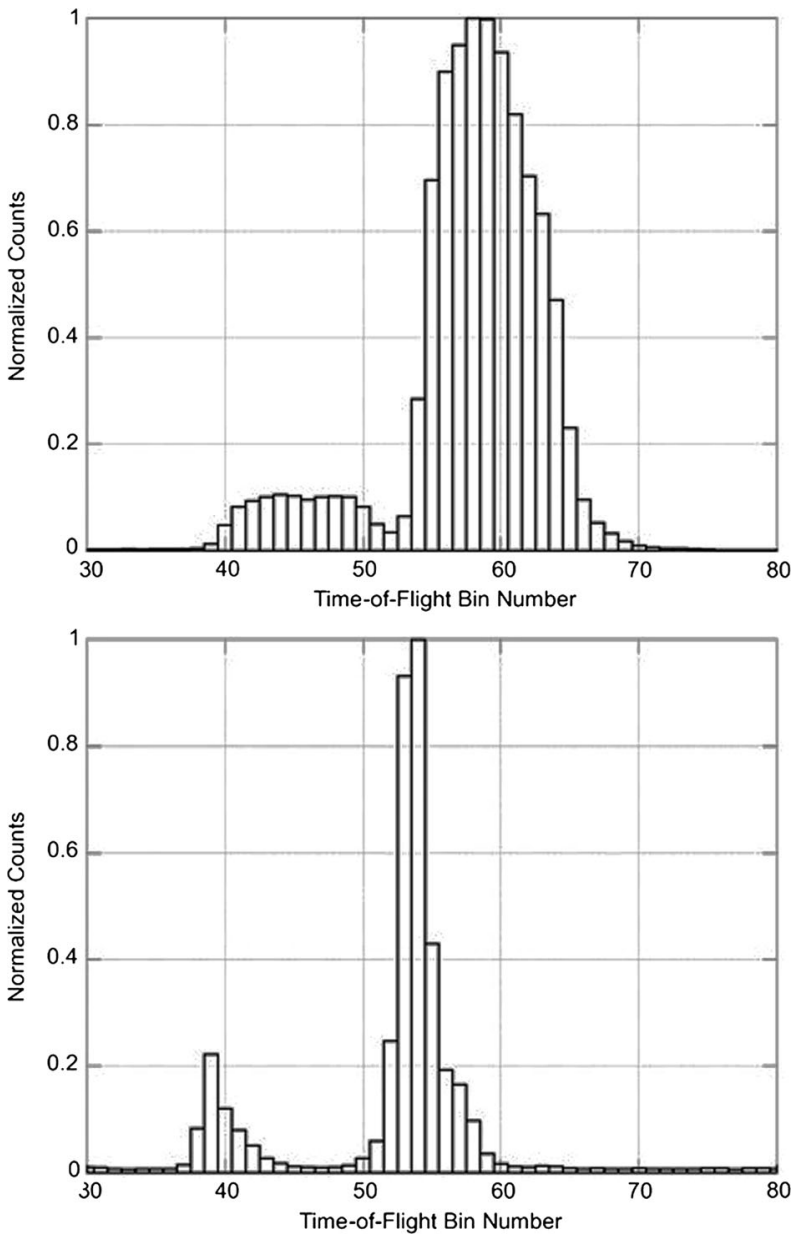

comprised of 4 components: the time of ion transit from the outboard edge of the TOF gate to the outer "dome", $\tau_{1}$; the corresponding time from the outer dome to the front of the MCP detector, $\tau_{2}$; the transit time of the electron charge cloud through the MCP to the anode pixel, $\tau_{3}$; and the time delay of the pre-amplifier firing, $\tau_{4}$.

For a given ion energy, $\tau_{1}$ is essentially proportional to the square root of the ion mass, and $\tau_{2}$ depends on both the ion mass and the magnitude of $\Delta V_{S A}$, and is typically small compared with $\tau_{1}\left(\tau_{2} / \tau_{1} \leq 0.1\right) ; \tau_{3}$ is on the order of $1 \mathrm{~ns}$ and therefore negligible compared with $\tau_{1}$ and $\tau_{2}$. The quantity $\tau_{4}$ is dominated by the triggering time of the preamplifiers, which is dependent on the rate of charge injection and the pulse amplitude of the injected charge, and was found to be about $220 \mathrm{~ns}$ under typical sensor operating conditions. Including the rise time of $\sim 25 \mathrm{~ns}$ and the fall time of $\sim 80 \mathrm{~ns}, \tau_{4}$ is about $320 \mathrm{~ns}$ or 8 TOF bins. Thus, the starting TOF bin number of a measured ion is approximately given by $\left(C_{1}(m / q)^{1 / 2}+C_{2}\right)$, where the coefficient $C_{1}$ depends on $\Delta V_{S A}$ and the ion energy-percharge, the coefficient $C_{2} \approx 8$, and $m / q$ is ion mass-per-charge. In the case of Fig. $10, C_{1}$ is approximately 8.9 (AMU/q) $)^{-1 / 2}$.

The discernible difference in TOF peak width between the top and bottom panels in Fig. 10 is attributed to the different TOF gate-open period in each case. The TOF gate elec- 
trode has a width of $0.6 \mathrm{~mm}$, and therefore takes about $0.038 \mu$ s for a $50-\mathrm{eV} \mathrm{N} \mathrm{N}_{2}^{+}$ion (and about $0.027 \mu$ s for a corresponding $\mathrm{N}^{+}$ion) to traverse. Therefore, in the top panels, all of the ions arriving at the TOF gate within the first 9 of the 10 TOF bins after the gate opening (i.e. the first $0.36 \mu$ s of the $0.40 \mu$ s gate-open duration) will have sufficient time to traverse the electrode before the gate closes.

In contrast, in the bottom panel, the TOF gate-open duration of $0.04 \mu$ s is comparable to the transit time of the $\mathrm{N}_{2}^{+}$ions through the TOF gate electrode, and a substantial fraction of ions arriving at the TOF gate during the gate open period failed to exit the TOF gate before the end of the gate-open period. This explains the relatively flat peaks spanning several TOF bins in the top panel and the much narrower TOF peaks in the bottom panel. It also means that the TOF gate must remain open for at least 4 or 5 TOF bin periods for measurements of cold ions on orbit, which typically have a velocity of $\sim 8 \mathrm{~km} / \mathrm{s}$ in the sensor frame of reference and require about $0.1 \mu$ s to traverse through the TOF gate.

The detailed response function of the sensor at each energy pixel varies as a function of ion energy $E$. This includes the geometric factor $G(E, \Omega)$, the energy resolution $(\Delta E / E)$ and angle of acceptance $(\Delta \Omega)$. As noted above, a particle-tracing code was used to compute the electric field distribution inside the sensor and in the vicinity of its entrance aperture as a function of sensor voltage settings, and to simulate the ion trajectories inside the sensor as a function of incident ion energy-per-charge, mass-per-charge, elevation and azimuth at the respective sensor voltage settings. The simulated trajectories were then used to simulate the sensor response, for comparison with the laboratory calibration measurements.

In azimuth and elevation calibration runs, the sensor elevation and azimuth were varied over a range of $\pm 5^{\circ}$ and $\pm 45^{\circ}$, respectively, to confirm the sensor's angle of acceptance in elevation and its varying angle of acceptance in azimuth with detector pixel radius. In sensitivity calibration runs, $V_{C P}$ was varied over its operational range, to characterize the dependence of ion detection sensitivity on the MCP front surface bias. In TOF calibration runs, the measured ion TOF distribution at a selected energy pixel and $N_{O P N}$ value was compared with the corresponding simulated distribution, in terms of the starting and peak location, width, and shape of the TOF peak. The two distributions were found to be typically within $1-2 \%$ of each other.

In energy calibration runs, the sensor stepped through a wide range of $V_{S A}$ values, to determine the ranges of $V_{S A}$ values in which ions of a given energy-per-charge reach a selected energy pixel and its neighboring energy pixels, respectively, for comparison with the simulated ranges of $V_{S A}$ values. The two ranges were found to generally agree to within $\sim 3-5 \%$. The combination of TOF and energy calibration makes it possible to characterize the energy-per-charge of the measured ions precisely $(\sim 2-4 \%)$, despite the large energy width of individual energy pixels (cf. Fig. 6).

Based on the generally good agreement (a few percent) in both energy and TOF response, the simulated sensor response will be used for nominal conversion of observed ion count data to (approximate) differential ion flux and other geophysical quantities. For accurate determination of detailed velocity phase space density distribution, an iterative approach taking into account the dependence of the overall sensor response on the incident ion distribution is planned, and is beyond the scope of this report.

\section{Initial Observations}

Figure 11a, 11b, 12 and 13a, 13b, 14 illustrate typical features in the observed IRM data at low and high altitudes, respectively, and the variability of these features in the first year 
Fig. 11a Observed IRM data in a near-perigee pass at 11:56:53-12:06:51 UT on May 4, 2014: energy-angle versus time (EA-t) spectrogram of averaged ion count rate in the order of pixel sectors and pixel radius within a pixel sector, at $V_{S A}=-61,-149,-236$, and $-348 \mathrm{~V}$ (top to bottom)

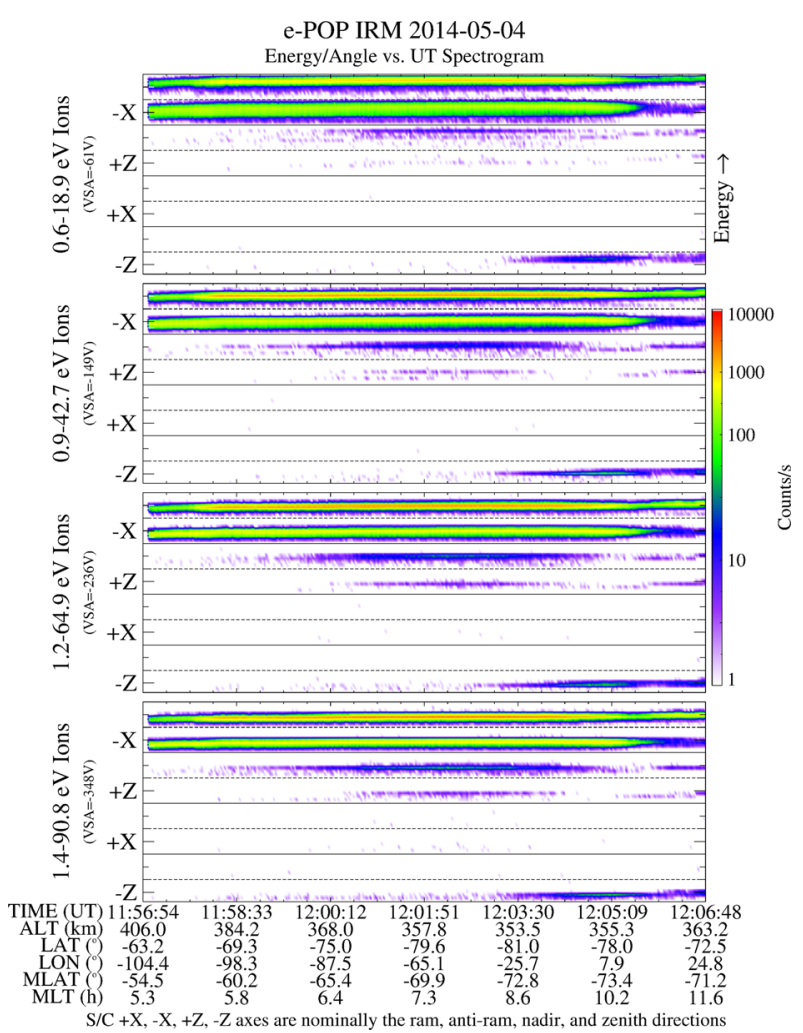

of e-POP operation. Detailed case and statistical analyses are in progress to investigate the underlying physics of these features in the context of topside ionospheric ion composition and outflow.

The IRM instrument is capable of operating in pre-programmed "measurement modes" tailored to supporting specific science investigations on orbit, in response to ground command. These include the "fast mode", in which all sensor voltages including $V_{S A}$ and $V_{E D}$ are kept fixed; the "survey mode", in which the sensor cycles through multiple $V_{S A}$ steps while keeping the other sensor voltages and the TOF gate timing parameters unchanged in order to survey the energy space more fully; and the "3D scan mode", in which the sensor cycles through multiple $V_{E D}$ steps $\left(N_{E D}\right)$ and optionally multiple $V_{S A}$ steps within each $V_{E D}$ step to obtain a 3D scan of the incident ion distribution.

In each measurement mode, the sensor voltage settings remain fixed in a measurement period, and each energy-angle pixel samples ions of a fixed incident azimuth and elevation, and range of ion energy-per-charge. At the end of a measurement, the energy-angle pixel and TOF data words in the FIFO are packetized into a data packet together with instrument status, housekeeping, and analog monitor data, including the measured sensor skin current during the period. A data packet can contain up to a maximum of 32,768 data words $(65,536$ bytes).

In the first year of IRM instrument operation, the number of TOF cycles $N_{T O F}$ in a measurement was set to a default value of 400 , corresponding to a measurement period of $16 \mathrm{~ms}$, and the gate open period was set to 11 TOF bin periods (i.e. $N_{O P N}=11$ ), or $0.44 \mu$ s. The 
Fig. 11b Observed IRM data in a near-perigee pass at 11:56:53-12:06:51 UT on May 4, 2014: time-of-flight versus time (TOF-t) spectrogram of the ion count rate, at $V_{S A}=-61,-149$, -236 , and $-348 \mathrm{~V}$ (top to bottom)

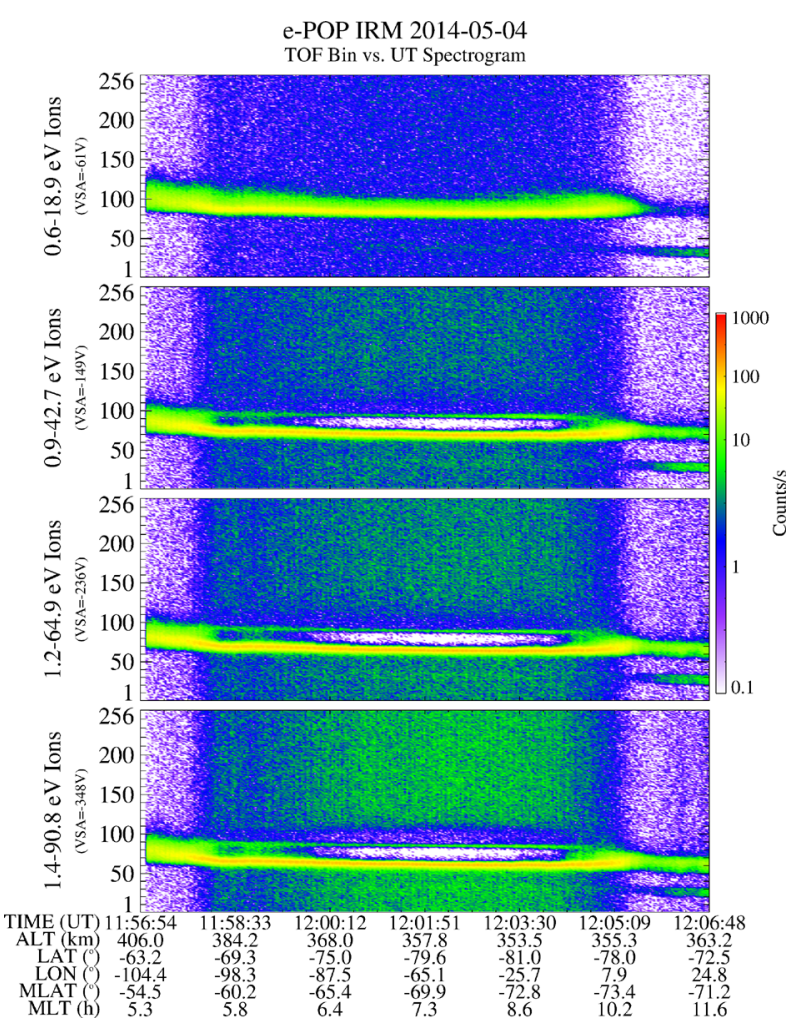

e-POP IRM 2014-05-04

survey mode was used predominantly, typically in a triangular stepping sequence of 16 or $12 V_{S A}$ steps, with a complete measurement cycle of 256 or $192 \mathrm{~ms}$, respectively.

Figures $11 \mathrm{a}, 11 \mathrm{~b}$ and 12 present the observed IRM data in a quiet-time $(K p=2-)$ morning sector (5.3-11.6 MLT) near-perigee pass at 11:56:54-12:06:48 UT on May 4, 2014. At this time, CASSIOPE was below $406 \mathrm{~km}$ altitude and IRM was operating in a survey mode. Figure $11 \mathrm{a}$ and $11 \mathrm{~b}$ show the energy-angle versus time (EA-t) and TOF versus time (TOF-t) spectrograms, respectively, of the observed ion count rate in this pass. Figure 12 shows the 1min averaged count rate in each (a) energy-angle pixel and (b) TOF bin at 12:01:30 near the middle of the pass. In both figures $\left|V_{S A}\right|$ increases from top to bottom: $V_{S A}=-62,-149$, -237 , and $-348 \mathrm{~V}$, corresponding to sampled ion energy-per-charge ranges of 0.6-18.9, 0.9-42.7, 1.2-64.9, and 1.4-90.8 eV/q, respectively.

As shown in Fig. 1a, the $+X$ and $+Z$ pixel sectors of the IRM sensor are aligned along the spacecraft $X$ and $Z$ axis, which are nominally in the spacecraft velocity and nadir direction, respectively, so that the $-\mathrm{X}$ and $-\mathrm{Z}$ sectors nominally sample ions from the spacecraft ram direction and those from below the spacecraft, respectively. In this orbit pass, the spacecraft anti-ram direction is within $5^{\circ}$ anticlockwise from the $-\mathrm{X}$ pixel sector, i.e. $<5^{\circ}$ toward the $-\mathrm{X}-\mathrm{Z}$ sector, and the upward magnetic field direction is $\sim 10^{\circ}$ clockwise from the $-\mathrm{Z}$ pixel sector, i.e. $\sim 10^{\circ}$ toward the $-\mathrm{X}-\mathrm{Z}$ sector.

In Figs. 11a and 12a, the averaged ion count rate is displayed in the order of pixel sector and the order of increasing pixel radius (and hence ion energy-per-charge) within each sector, starting from the $-\mathrm{Z}$ sector at the bottom in Fig. 11a and at the left in Fig. 12a, respectively. In Fig. 12a, the observed ion count rate in each energy-angle pixel is summed over all TOF bins, and each of the energy pixels is color coded. 


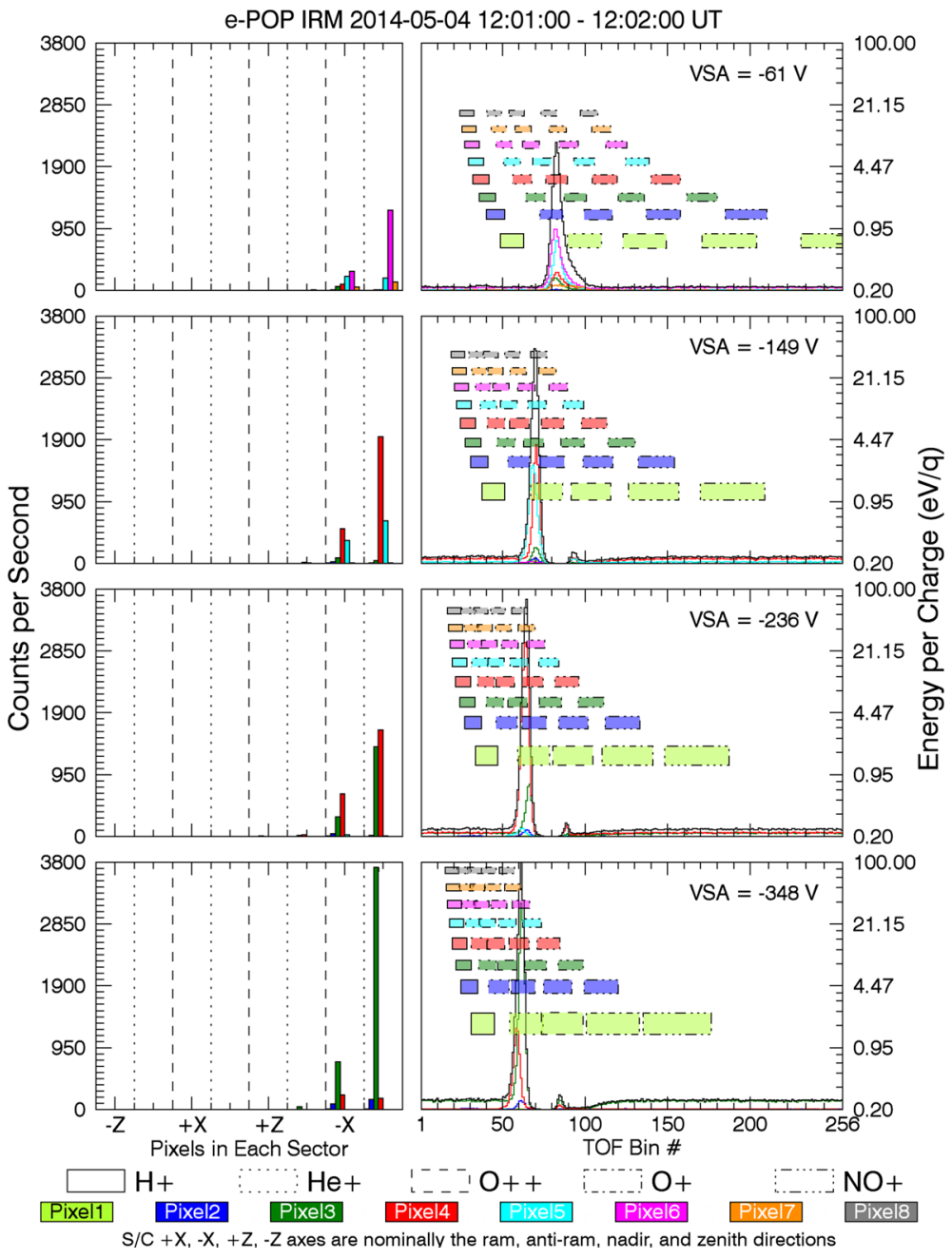

Fig. 12 IRM data on May 4, 2014. (a) (Left) Ion count rate versus detector pixel in each pixel sector; (b) (right) ion count rate in each detector pixel (color traces) and total ion count rate (black trace) and $\mathrm{H}^{+}$, $\mathrm{He}^{+}, \mathrm{O}^{++}, \mathrm{O}^{+}$and $\mathrm{NO}^{+}$ion energy versus TOF bin number, for $V_{S A}=-61,-149,-236$, and $-348 \mathrm{~V}$ (top to bottom)

The observed ion count rate was highest in the $-\mathrm{X}-\mathrm{Z}$ sector and second highest in the $-\mathrm{X}$ sector at all four $V_{S A}$ settings throughout the orbit pass, meaning ions arriving predominantly from below the spacecraft ram $(+X+Z)$ direction and from the spacecraft ram $(+X)$ direction, respectively. In comparison, the count rate in the $-\mathrm{X}+\mathrm{Z}$ and $+\mathrm{Z}$ pixel sectors was 2-3 orders of magnitude lower, suggesting that the peak of the incident ion distribution was closer to the $-\mathrm{X}-\mathrm{Z}$ than the $-\mathrm{X}+\mathrm{Z}$ direction.

In both sectors, there is a noticeable inward shift in the peak energy pixel to a more innermost (lower) energy pixel as $\left|V_{S A}\right|$ increases. In Fig. 12, the averaged ion count rate peaks in the $-\mathrm{X}-\mathrm{Z}$ pixel sector at all $4 V_{S A}$ settings. The peak pixel in this sector shifts inward as $\left|V_{S A}\right|$ increases, from the third outermost (highest) energy pixel (pixel 6, in magenta) in the 
top panel to the third innermost (lowest) energy pixel (pixel 3, in green) in the bottom panel, consistent with ions of a given energy being accelerated by a larger $\Delta V_{S A}$ to a higher energy and deflected onto a more innermost (lower) energy pixel at larger $\left|V_{S A}\right|$. The different count rate ratio between the $-\mathrm{X}-\mathrm{Z}$ and the $-\mathrm{X}$ sector in the different $V_{S A}$ panels reflects the fact the measured ion distribution peaked toward the $-\mathrm{X}-\mathrm{Z}$ sector, and each energy pixel in the two sectors in general sampled a different angular fraction of the incident ion distribution.

Figures $11 \mathrm{~b}$ and $12 \mathrm{~b}$ show the averaged ion count rate as a function of TOF. In Fig. 12b, the observed count rate in each energy pixel is summed over all pixel sectors (color traces and left axis). The color triangles in Fig. 12b denote the ion energy-per-charge and TOF ranges of each pixel. Each major division on the right axis corresponds to a factor of 4.7 increase in energy-per-charge, and the different border line styles correspond to different ion mass species: solid, dot, dash, dot-dash, and dot-dot-dot-dash for $\mathrm{H}^{+}, \mathrm{He}^{+}, \mathrm{O}^{++}, \mathrm{O}^{+}$ and $\mathrm{NO}^{+}$ions, respectively; the rectangles for $\mathrm{N}^{+}$and for $\mathrm{O}_{2}^{+}$and $\mathrm{N}_{2}^{+}$are not shown as their TOF ranges overlap with those for $\mathrm{O}^{+}$and for $\mathrm{NO}^{+}$, respectively; cf. Fig. 6 .

In Fig. 11b, a prominent TOF peak is present between 11:56:57 and 12:05:15, and the location of this peak shifts toward smaller TOF values as $\left|V_{S A}\right|$ increases, from a TOF range of $\sim 85-95$ at $V_{S A}=-62 \mathrm{~V}$ in the top panel to a range of $\sim 55-65$ at $V_{S A}=-348 \mathrm{~V}$ in the bottom panel. This shift reflects the fact that the observed ions arrived at the detector at earlier arrival times at larger $\left|V_{S A}\right|$, as discussed earlier. In each $V_{S A}$ panel, a shift toward smaller ion TOF in the middle of the orbit pass is discernible; this shift is consistent with a corresponding, outward shift in the peak energy pixel to a higher (more outermost) energy pixel in Fig. 11a. A secondary and narrower peak is also present at TOF bin $\sim 100$ between 11:58:14 and 12:04:58, except in the top panel. As will be discussed below, this secondary peak exhibits a similar shift toward smaller TOF values, from TOF bin $~ 93$ in the second top panel to 87 and 84 in the two bottom panels as $\left|V_{S A}\right|$ increases from 149 to 237 and $348 \mathrm{~V}$, respectively.

Figure $12 \mathrm{~b}$ shows that the peak of the total ion count rate (black trace) shifts from TOF bin of $\sim 81$ to $\sim 70,65$, and 62 as $\left|V_{S A}\right|$ increases from 62 to 149,237 , and $348 \mathrm{~V}$, respectively. In each $V_{S A}$ panel, the ion count rate at the peak energy pixel starts to increase from its background value within a few TOF bins to the left of the expected TOF range for $\mathrm{O}^{+}$, as indicated by the magenta, red, red and green, and green dot-dash rectangles in the respective $\left|V_{S A}\right|$ panels. This indicates the presence of $\mathrm{O}^{+}$ions as the dominant ion species and $\mathrm{N}^{+}$ions in lower fluxes, in the energy range of 7.1-8.5, 6.0-7.6, 8.5-11.1, and 6.7-8.5 eV/q in the respective panels, since as noted above, the TOF range of $\mathrm{N}^{+}$ions is adjacent to or overlaps with that of $\mathrm{O}^{+}$for a given ion energy.

In the top $\left(V_{S A}=-62 \mathrm{~V}\right)$ panel, the $\mathrm{O}^{+}$ions were observed in TOF bin 84-88, energy pixel 6 (magenta trace and dot-dash rectangle), and in TOF bin 93-98, pixel 5 (light blue trace and dot-dash rectangle). The $\mathrm{N}^{+}$ions were observed in TOF bin 79-83, energy pixel 6, and in TOF bin 88-92, pixel 5 (magenta and blue trace, respectively; rectangles not shown for clarity). In addition to the $\mathrm{O}^{+}$and $\mathrm{N}^{+}$ions, the observed ions also comprised a smaller component of $\mathrm{O}^{++}$, in TOF bin 76-81, energy pixel 4 (red trace) and in TOF bin 87-93, pixel 3 (green trace). Note that for the same ion energy, $\mathrm{O}^{++}$ions being doubly charged have half the ion energy-per-charge of $\mathrm{O}^{+}$ions. This explains their detection in energy pixel 3 and 4, which sample the 1.9-2.3 and 2.9-3.7 eV/q range at $V_{S A}=-62 \mathrm{~V}$, instead of pixel 5 and 6, which sample the 4.6-5.6 and 7.1-8.5 eV/q range, respectively.

The observed $\mathrm{N}^{+}$ions are evident in the three larger $\left|V_{S A}\right|$ panels as well, for example at $V_{S A}=-149 \mathrm{~V}$ in TOF bin 61-64, energy pixel 5, in TOF bin 69-74, pixel 4, and in TOF bin $80-86$, pixel 3 (light blue, red, and green trace left of the dot-dash rectangle of the same color, respectively). Likewise, the observed $\mathrm{O}^{++}$ions are evident in TOF bin 62-67, energy pixel 3, and TOF bin 72-79, pixel 2 (green and blue traces and dot rectangles). 
As noted above, a secondary TOF peak is present in the three larger $\left|V_{S A}\right|$ panels. Figure $12 \mathrm{~b}$ shows that this secondary peak shifts from TOF bin $\sim 93$ to 87 and 84 as $\left|V_{S A}\right|$ increases from 149 to 237 and $348 \mathrm{~V}$, and corresponds to $\mathrm{NO}^{+}$ions observed in energy pixel 5, 4, and 3 (light blue, red, and green trace and rectangle), respectively. Based on the ion energy versus TOF relationship in Fig. 6, the observed ion count rates and pixel locations in these three $V_{S A}$ panels signify the presence of $\mathrm{NO}^{+}$in the energy range of 9.8 to 11.9 , 8.6 to 11.0 , and $\sim 8.3$ to $\sim 11.3 \mathrm{eV}$, respectively. This explains the absence of a secondary TOF peak in the top panel, since at $V_{S A}=-62 \mathrm{~V}$, the majority of the observed ions (those between 8.5 and $10.6 \mathrm{eV} / \mathrm{q}$ ) would land between energy pixel 6 and 7 on the detector and would not be detected by either one. In comparison, the spacecraft ram energy of $\mathrm{NO}^{+}$ions was $\sim 7.5 \mathrm{eV}$. In other words, the ions have an energy of $\sim 1$ to a few $\mathrm{eV}$.

The observed $\mathrm{NO}^{+} / \mathrm{O}^{+}$peak ion count ratio was $\sim 0.05$ in this orbit pass, and appears to be quite variable in other low-altitude orbit passes where $\mathrm{NO}^{+}$was observed. In comparison, the International Reference Ionosphere (IRI) model (Bilitza and Reinisch 2008), for example, predicts a negligible $\mathrm{NO}^{+} / \mathrm{O}^{+}$density, less than $1 \%$ above the F-region peak in the daytime mid-latitude ionosphere. The variability of molecular ion composition in the F-region and topside ionosphere and their possible role as a source of energetic molecular ions in the magnetosphere will be investigated in a planned future study.

A non-negligible (few counts/s) background ion count rate is apparent in the TOF-t spectrograms between $\sim 11: 56: 57$ and 12:05:10. Figure $12 \mathrm{~b}$ shows that the background rate in each TOF bin is roughly proportional to the corresponding contribution to the peak ion count rate. This background rate is attributed to ions arriving at the TOF gate during the closed period of the TOF cycle. Such ions are deflected onto the top TOF electrode but a statistically small fraction (presumably $<10 \%$ ) of them could be scattered back onto the TOF gate after the first one or two deflections, and eventually reach the detector, resulting in the background level observed in Figs. 11a, 11b.

Figures 13a and 13b present the EA-t and TOF-t spectrograms, respectively, of observed data in a quiet-time $(K p=1+)$ post-midnight sector (1.1-6.1 MLT) high-altitude (1107$1275 \mathrm{~km}$ ) pass at 10:24:03-10:30:11 UT on April 9, 2014, in the same data format as in Fig. 11a, 11b. Figure 14 shows the 1-min averaged ion count rates centered at 10:25:00, in the same data format as in Fig. 12. In this orbit pass, the spacecraft ram direction is within $5^{\circ}$ clockwise from the $-\mathrm{X}$ pixel sector, i.e. $<5^{\circ}$ toward the $-\mathrm{X}+\mathrm{Z}$ sector, and the upward magnetic field direction is aligned with the $-\mathrm{Z}$ pixel sector.

Figures 13a, 13b shows that as in the May 4 orbit pass in Figs. 11a, 11b: (i) The observed TOF peak shifts toward smaller TOF bin number with increasing $\left|V_{S A}\right|$; (ii) In each $V_{S A}$ panel, the peak count rate at the innermost (lowest) energy pixels peaks at larger TOF bin number, reflecting the lower ion energy and longer TOF; and (iii) Within the primary TOF peak, the observed ions in the different energy pixels in general comprise multiple ion species, including $\mathrm{O}^{+}, \mathrm{N}^{+}$, and $\mathrm{O}^{++}$. In comparison with Fig. 11a, 11b: (iv) The observed ion count rate in this orbit pass peaked in the $-X$ sector, and was non-negligible in the $-\mathrm{X}+\mathrm{Z}$ sector; (v) The observed ion count rate peaked at a more innermost (lower) energy pixel at a given $V_{S A}$; (vi) Likewise, the primary TOF distribution peaked at a higher TOF bin compared with the value of $\sim 82$ in Fig. 12, and increased from $\sim 92$ at the beginning of the pass $(10: 24: 20)$ to $\sim 98$ at the end of the pass $(10: 30: 10)$ in the top $\left(V_{S A}=-61 \mathrm{~V}\right)$ panel.

It is apparent in Fig. 14 that despite the broad pixel energy response $(\Delta E / E$ ranges from 5-7 \% to 15-24\%; cf. Fig. 6), it is possible to derive the energy distribution of each ion species to a better precision from the observed TOF distributions in the different energy pixels (color traces) at different $V_{S A}$. For example, at $V_{S A}=-148 \mathrm{~V}$ (second panel), the observed $\mathrm{O}^{+}$ion count rate peaked in energy pixel 4 (red trace) and the observed TOF 
Fig. 13a Observed IRM data in a near-apogee pass at 10:24:10-10:30:10 UT on April 9, 2014: energy-angle versus time (EA-t) spectrogram of averaged ion count rate in the order of pixel sectors and pixel radius within a pixel sector, at $V_{S A}=-62,-149,-236$, and $-348 \mathrm{~V}$ (top to bottom)

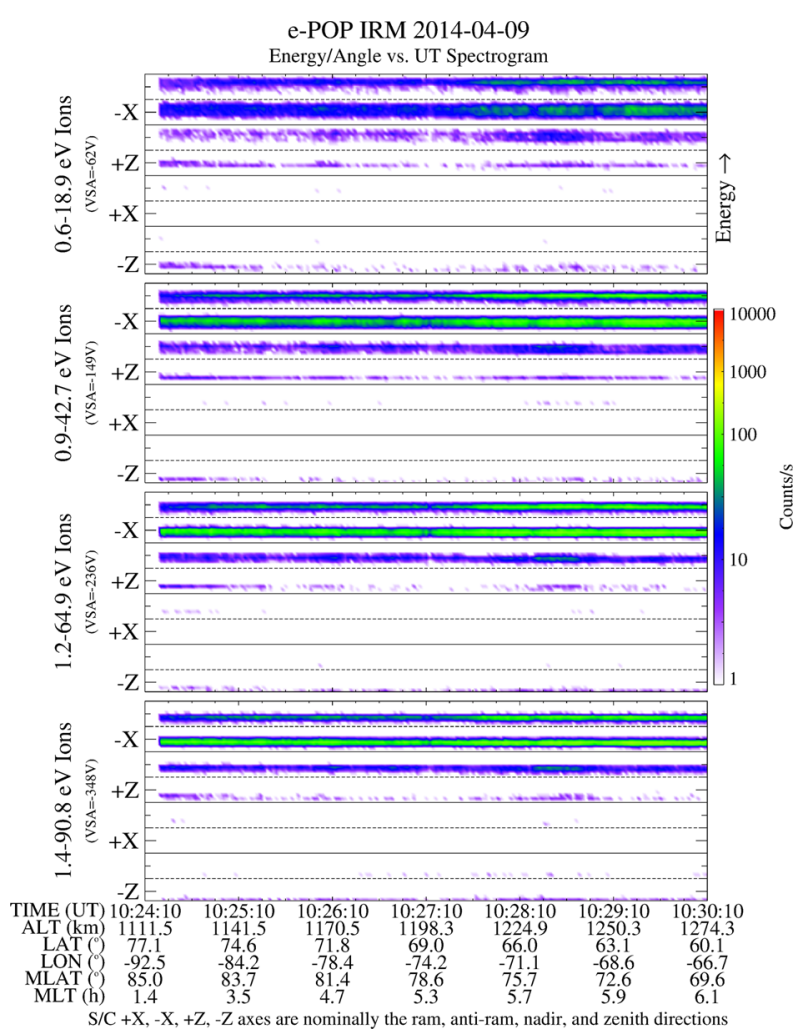

peak spanned the expected TOF range (red dot-dash rectangle). In contrast, the observed count rate was higher at the lower end of the expected TOF range in energy pixel 3 (green trace) and at the higher end of the range in pixel 5 (light blue trace). This signifies a peaked distribution at the higher energy end in pixel 3 and at the lower energy end in pixel 5, respectively, and a more uniform distribution in pixel 4.

The same pattern is evident at $V_{S A}=-62 \mathrm{~V}$ (top panel). The ion energy distribution of the observed $\mathrm{O}^{+}$ion count rate was uniform in energy pixel 5, and peaked at the higher and lower end of the ion energy range in pixel 4 and 6 , respectively. Both $\mathrm{H}^{+}$and $\mathrm{O}^{++}$ions were observed, but in energy pixel 2-4 (blue, green and red trace) due to their smaller energy-percharge compared with $\mathrm{O}^{+}$because of the lighter mass of $\mathrm{H}^{+}$and the double charge of $\mathrm{O}^{++}$. In both cases, the observed ion energy distribution in energy pixel 2 (i.e. the lower energy pixel) is seen to peak at the higher end of the ion energy-per-charge range within the pixel.

In Fig. 14, at $V_{S A}=-62 \mathrm{~V}$ (top panel), the observed $\mathrm{O}^{+}$ion count rate peaked in energy pixel 5 at 4.6-5.6 eV/q (light blue trace); within the adjacent pixels, the rate peaked at the higher energy end of pixel 4 (red) and the lower energy end of pixel 6 (magenta). Likewise, the rate for $\mathrm{N}^{+}, \mathrm{O}^{++}$and $\mathrm{H}^{+}$peaked in energy pixel 4 at 2.9-3.7 eV/q (red trace), pixel 3 at $1.9-2.3 \mathrm{eV} / \mathrm{q}$ (3.8-4.6 eV; green trace), and pixel 3 at $1.9-2.3 \mathrm{eV} / \mathrm{q}$ (green trace), respectively. A quantitative velocity analysis for the respective ion species requires a precise knowledge of the sensor potential, particularly for $\mathrm{H}^{+}$, and is beyond the scope of this report. However, assuming near-zero sensor floating potential, the observed ion energy-per-charge range of the other ion species corresponds to an ion velocity range of $6.3-9.6 \mathrm{~km} / \mathrm{s}$ for the 
Fig. 13b Observed IRM data in a near-apogee pass at 10:24:10-10:30:10 UT on April 9, 2014: time-of-flight versus time (TOF-t) spectrogram of the ion count rate, at $V_{S A}=-62$, $-149,-236$, and $-348 \mathrm{~V}$ (top to bottom)

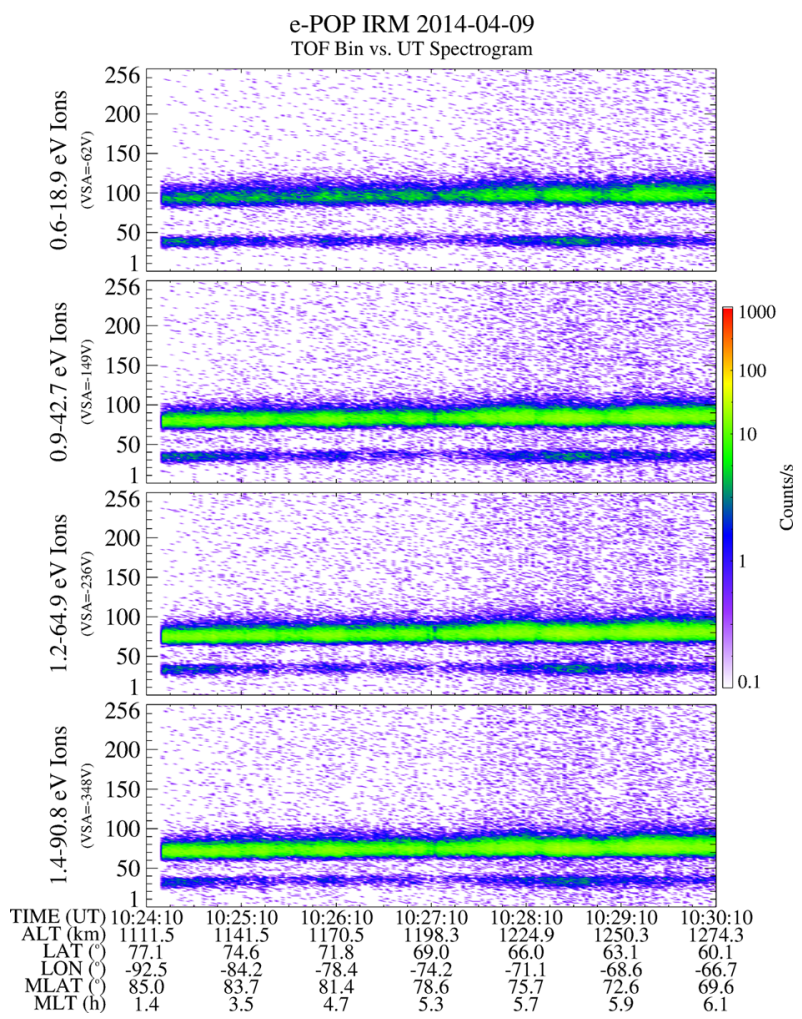

other species, compared with the spacecraft ram velocity of $\sim 7.2 \mathrm{~km} / \mathrm{s}$, suggesting that the observed ion energy for these species was dominated by spacecraft ram.

\section{Summary and Discussion}

The measurement objective of the imaging and rapid-scanning ion mass spectrometer (IRM) on the CASSIOPE spacecraft is to measure in-situ both the mass composition distribution of the thermal-energy $(\sim 1-100 \mathrm{eV} / \mathrm{q})$ plasma population in the topside ionosphere and the mass-resolved velocity phase space distribution of each major ion species, and to use the measured distributions to derive the bulk plasma parameters and to investigate the occurrence and dynamics of ion up-flows or outflows in the topside ionosphere.

The IRM uses a toroidal electrostatic deflector to sample ions at different elevations (angles to the sensor entrance aperture plane), a time-of-flight (TOF) gate to measure the individual ion TOF, and a hemispherical electrostatic analyzer (HEA) to determine the individual energy-per-charge and incident azimuth. However, the toroidal deflector was used for 3D survey only very sparingly to date, in order to avoid any risk of its operation affecting the quality of field and wave measurements on board in the first year of e-POP operation; therefore only TOF and HEA data are presented in this report.

The instrument is designed to measure thermal-energy ions in the energy-per-charge range of $\sim 1$ to $100 \mathrm{eV} / \mathrm{q}$ and the mass-per-charge range of 1 to $>40$ atomic mass units per charge (AMU/q) at up to $\sim 10 \%$ energy resolution $(\Delta E / E)$ and $\sim 5^{\circ}$ angular resolution, 


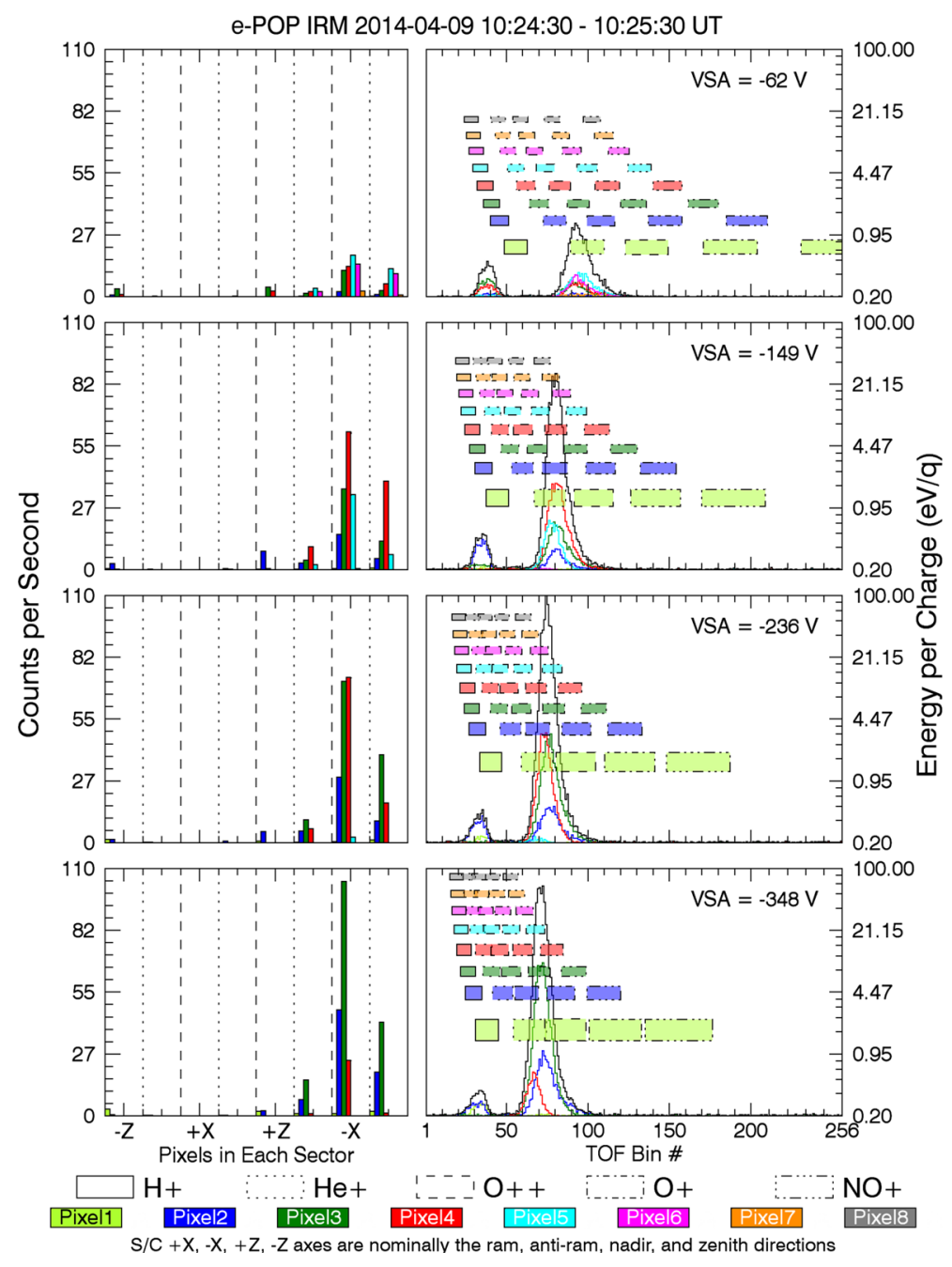

Fig. 14 IRM data on April 9, 2014. (a) (left) Ion count rate versus detector pixel in each pixel sector; (b) (right) ion count rate in each detector pixel (color traces) and total ion count rate (black trace) and $\mathrm{H}^{+}$, $\mathrm{He}^{+}, \mathrm{O}^{++}, \mathrm{O}^{+}$and $\mathrm{NO}^{+}$ion energy versus TOF bin number, for $V_{S A}=-62,-148,-236$, and $-348 \mathrm{~V}$ (top to bottom)

and to resolve all major ion species in the ionosphere including $\mathrm{H}^{+}, \mathrm{He}^{+}, \mathrm{O}^{+}$as well as adjacent molecular ion species such as $\mathrm{N}_{2}^{+}, \mathrm{NO}^{+}$and $\mathrm{O}_{2}^{+}$under favorable conditions.

In the preceding sections, Figs. 3-4 explain the principle of operation of the IRM sensor, and Figs. 5-6 and Figs. 9-10 describe its response. Figures 11a, 11b, 12, 13a, 13b, and 14 illustrate typical features in the observed IRM data at low and high altitudes, respectively, and the variability of these features in the first year of e-POP operation. Detailed case and statistical analyses are in progress to investigate the underlying physics of these features in the context of topside ionospheric ion composition and outflow. 
Compliance with Ethical Standards This work was funded by the Canadian Space Agency (CSA) under Contribution Agreement LS2003-002 and by the Natural Science and Engineering Research Canada (NSERC) under Discovery Grant RGPIN 06069. The authors declare that they have no conflict of interest.

Acknowledgement We gratefully acknowledge the funding support for the e-POP project from the Canadian Space Agency (CSA) and the Natural Science and Engineering Research Canada (NSERC). We also wish to thank the many individuals who contributed to the development and operation of the IRM, including Mark Astridge, Yi-Ching Chung, Robert Hum, Troy Kachor, E. Peter King, Clifford Marcellus, Jamie Roberts, and Ivan Wevers.

Open Access This article is distributed under the terms of the Creative Commons Attribution 4.0 International License (http://creativecommons.org/licenses/by/4.0/), which permits unrestricted use, distribution, and reproduction in any medium, provided you give appropriate credit to the original author(s) and the source, provide a link to the Creative Commons license, and indicate if changes were made.

\section{References}

T. Abe, B.A. Whalen, A.W. Yau, R.E. Horita, S. Watanabe, E. Sagawa, EXOS-D (Akebono) SMS observations of the polar wind. J. Geophys. Res. 98, 11191-11203 (1993)

M. Andre, P. Norqvist, L. Andersen, L. Eliasson, A.I. Eriksson, L. Blomberg, R.E. Erlandson, J. Waldemark, Ion energization mechanisms at 1700 kilometer in the auroral region. J. Geophys. Res. 103, 4199-4222 (1998)

D. Bilitza, B. Reinisch, International Reference Ionosphere 2007: improvements and new parameters. Adv. Space Res. 42(4), 599-609 (2008). doi:10.1016/j.asr.2007.07.048

W.H. Bennett, Radiofrequency mass spectrometer. J. Appl. Phys. 21(2), 143-149 (1950)

C.W. Carlson, D.W. Curtis, G. Paschmann, W. Michel, An instrument for rapidly measuring plasma distribution functions with high resolution. Adv. Space Res. 2(7), 67-70 (1983)

C.R. Chappell, S.A. Fields, C.R. Baugher et al., The retarding ion mass spectrometer on Dynamics Explorer-A. Space Sci. Instrum. 5, 477-491 (1981)

C.R. Chappell, The terrestrial plasma source: a new perspective in solar-terrestrial processes from Dynamics Explorer 1988. Rev. Geophys. 26(2). doi:10.1029/RG026i002p00229

L.L. Cogger, A. Howarth, A.W. Yau, A. White, G. Enno, T. Trondsen, D. Asquin, B. Gordon, P. Marchand, D. Ng, G. Burley, M. Lessard, B. Sadler, Fast Auroral Imager (FAI) for the e-POP mission. Space Sci. Rev. (2014, this issue). doi:10.1007/s11214-014-0107-x

W.R. Coley, R.A. Heelis, M.R. Hairston, G.D. Earle, M.D. Perdue, R.A. Power, L.L. Harmon, B.J. Holt, C.R. Lippincott, Ion temperature and density relationships measured by CINDI from the C/NOFS spacecraft during solar minimum. J. Geophys. Res. 115, A02313 (2010). doi:10.1029/2009JA014665

E. Engwall, A.I. Eriksson, C.M. Cully, M. Andre, R. Torbert, H. Vaith, Earth's ionospheric outflow dominated by hidden cold plasma. Nat. Geosci. (2009). doi:10.1038/NGEO387

W.B. Hanson, S. Sanatani, D.R. Zuccaro, The Martian ionosphere as observed by the Viking retarding potential analyzers. J. Geophys. Res. 82, 4351-4363 (1977)

H.G. James, E.P. King, A. White, R.H. Hum, W.H.H.L. Lunscher, C.L. Siefring, The e-POP radio receiver instrument on CASSIOPE. Space Sci. Rev. (2014, this issue). doi:10.1007/s11214-014-0130-y

D.J. Knudsen, J.K. Burchill, T.G. Cameron, G.A. Enno, A. Howarth, A.W. Yau, The CASSIOPE/e-POP Suprathermal Electron Imager (SEI). Space Sci. Rev. (2015, this issue)

R.L. Lysak, W. Lotko, On the kinetic dispersion relation for shear Alfven waves. J. Geophys. Res. 101(A3), 5085-5094 (1996)

M. Morooka, T. Mukai, Density as a controlling factor for seasonal and altitudinal variations of the auroral particle acceleration region. J. Geophys. Res. 108(A7), 1306 (2003). doi:10.1029/2002JA009786

C.J. Pollock, V.N. Coffey, J.D. England, N.G. Martinez, M.L. Adrian, Thermal electron capped hemisphere spectrometer (TECHS) for ionospheric studies, in Measurement Techniques in Space Plasmas: Particles, ed. by R.F. Pfaff, J.E. Borovsky, D.T. Young. Geophysical Monograph, vol. 102 (American Geophysical Union, Washington, 1998), p. 201

R.J. Redmon, W.K. Peterson, L. Andersson, W.F. Denig, A global comparison of $\mathrm{O}^{+}$upward flows at $850 \mathrm{~km}$ and outflow rates at $6000 \mathrm{~km}$ during nonstorm times. J. Geophys. Res. 117, A04213 (2012). doi:10.1029/2011JA017390 
R.J. Strangeway, R.E. Ergun, Y.J. Su, C.W. Carlson, R.C. Elphic, Factors controlling ionospheric outflows as observed at intermediate altitudes. J. Geophys. Res. 110, A03221 (2005). doi:10.1029/2004JA010829

D.D. Wallis, D.M. Miles, B.B. Narod, J.R. Bennest, K.R. Murphy, I.R. Mann, A.W. Yau, The CASSIOPE/e-POP Magnetic Field Instrument (MGF). Space Sci. Rev. (2014, this issue). doi:10.1007/ s11204-014-0105-Z

B.A. Whalen, J.R. Burrows, A.W. Yau et al., The Suprathermal Ion Mass Spectrometer (SMS) onboard the Akebono (EXOS-D) satellite. J. Geomagn. Geoelectr. 42, 511-536 (1990)

B.A. Whalen, D.J. Knudsen, A.W. Yau et al., The Freja F3C cold plasma analyzer. Space Sci. Rev. 70, 541561 (1994)

A.W. Yau, E. Drakou, M.J. Greffen, D.J. Knudsen, E. Sagawa, Radio-frequency ion mass spectrometer measurements of thermal ion composition, velocity, and temperature: the EXOS-D Suprathermal Mass Spectrometer, in Measurement Techniques in Space Plasmas: Particles, ed. by R.F. Pfaff, J.E. Borovsky, D.T. Young. Geophysical Monograph, vol. 102 (American Geophysical Union, Washington, 1998a), pp. 307312

A.W. Yau, G.P. Garbe, M.J. Greffen, W. Miyake, E. Sagawa, Planned observations of thermal plasma drifts and solar wind interactions in the Martian ionosphere. Earth Planets Sun 50, 195-198 (1998b) 